\title{
Structure shapes the representation of a novel category
}

\author{
Sarah H. Solomon* \& Anna C. Schapiro \\ Department of Psychology, University of Pennsylvania
}

* Author for correspondence: sarahsol@sas.upenn.edu

\begin{abstract}
Concepts contain rich structures that support flexible semantic cognition. These structures can be characterized by patterns of feature covariation: certain clusters of features tend to occur in the same items (e.g., feathers, wings, can fly). Existing computational models demonstrate how this kind of structure can be leveraged to slowly learn the distinctions between categories, on developmental timescales. It is not clear whether and how we leverage feature structure to quickly learn a novel category. We thus investigated how the internal structure of a new category is extracted from experience and what kinds of representations guide this learning. We predicted that humans can leverage feature clusters within an individual category to benefit learning and that this relies on the rapid formation of distributed representations. Novel categories were designed with patterns of feature associations determined by carefully constructed graph structures (Modular, Random, and Lattice). In Experiment 1, a feature inference task using verbal stimuli revealed that Modular categories — containing clusters of reliably covarying features-were more easily learned than nonModular categories. Experiment 2 replicated this effect using visual categories. In Experiment 3, a temporal statistical learning paradigm revealed that this Modular benefit persisted even when category structure was incidental to the task. We found that a neural network model employing distributed representations was able to account for the effects, whereas prototype and exemplar models could not. The findings constrain theories of category learning and of structure learning more broadly, suggesting that humans quickly form distributed representations that reflect coherent feature structure.
\end{abstract}




\section{Introduction}

Mental representations are only useful insofar as they capture the content and structure of the environment. Structure-the organization of relations among units in a system-can reflect different kinds of relations in different domains. For example, structure within the visual domain may reflect co-occurrences of visual features across scenes, spatial structure may reflect the physical relationships between various landmarks, and semantic structure may reflect the pattern of semantic feature associations on multiple conceptual scales. Recently, in part inspired by the idea of "cognitive maps" (Tolman, 1948), researchers have sought to understand the formation of structural representations and their influence on human cognition. Here we focus on the semantic domain, and the featurebased structure that underlies concepts and categories. Our approach is to synthesize ideas from structure learning and category learning to investigate the formation and nature of structured category representations.

We use the term concept to reflect the internal representation of a category, which is a set of ideas or items in the external world (Komatsu, 1992; Solomon et al., 1999; Rips et al. 2012). Research on concepts typically focuses on the rich, mature representations humans already carry with them around the world (e.g., FIREFLY, ROSE, TRUTH). On the other hand, research on category learning typically focuses on newly constructed categories defined by abstract perceptual stimuli (e.g., visual shapes, auditory tones) or simplified figures (e.g., cartoon animals, line drawings), lacking the richness in structure that eventually supports our mature concept representations. Here we use a category learning paradigm to target fundamental questions of concept representation-when a new category is learned, how can the initially formed representations lead to the rich, structured concepts that compose semantic memory?

\section{Structure within and between concepts}

Structure within the semantic domain can explain various aspects of semantic cognition, including the developmental trajectory of semantic learning (Rogers \& McClelland, 2004; Unger et al., 2020), performance on conceptual tasks such as property verification and similarity judgments (Rosch, 1975; McRae et al., 1997; 1999; Cree et al., 1999; Tyler et al., 2000), and patterns of flexible language use 
(Solomon et al., 2019). Classic studies suggest that concepts are not represented as collections of independent features, but as the "web of relationships in which these properties participate" (Medin et al., 1987). For example, one study found that participants considered material and size dimensions to be correlated within the SPOON concept: wooden spoons are typically large, and metal spoons are typically small (Medin \& Shoben, 1988). This was interpreted to suggest that semantic features are not independent but are structured via associative relations. Classic empirical findings on the interpretation of combined concepts (e.g., METAL ROSE) are often used to support the claim that concepts contain structured sets of features and/or relations (Wisniewski \& Gentner, 1991; Sloman et al., 1998).

Feature-based models of conceptual representation have highlighted the importance of correlational feature structure within and across semantic domains. For example, the Conceptual Structure account presented by Tyler and colleagues provides a framework for understanding why people consider certain kinds of features (i.e., functional vs. perceptual) as more important for some concepts versus others (i.e., artifacts vs. living things; Tyler et al., 1999; Tyler \& Moss, 2000). Work by McRae and colleagues similarly reveals how feature correlations predict performance on property verification, similarity judgment, and typicality judgment tasks (McRae et al., 1997; 1999; Cree et al., 1999). The structure of individual concepts also appears to have consequences for how those concepts are flexibly used in language. For example, in recent work we found evidence that the feature-based structure of a concept predicts its semantic variability as measured within language corpora (Solomon et al., 2019).

Rogers \& McClelland (2004) demonstrate that the structure of the semantic environment-that is, patterns of semantic feature co-occurrences across items-can be used by neural network models to learn hierarchical semantic relationships. The models build representations that differentiate superordinate categories (e.g., ANIMALS vs. PLANTS), basic-level categories (e.g., FLOWER vs. TREE), and individual items (e.g., ROSE vs. PINE TREE) in a manner that accounts for patterns of semantic cognition in humans: For example, the order in which concepts are learnt, the special status of basic level concepts, and the acquisition and deterioration of semantic knowledge observed in infants and dementia patients (Rogers \& McClelland, 2004). An important principle underlying the success of the models is that they leverage "coherent covariation" among clusters of features to build internal representations that capture the structure of the semantic environment (McClelland \& Rogers, 2003; Rogers \& McClelland, 2004; Saxe et al., 2019; Cree et al., 1999). Coherent covariation refers to the tendency of features to co-occur across different semantic items. For example, the presence of feathers 
and wings and the capability of flight are features that tend to co-occur, facilitating the formation of a BIRD concept.

Structure within the semantic domain thus appears relevant to concept acquisition, representation, and use. However, we do not yet understand how humans learn this rich semantic structure and what kind of representations are built in the process. One of the goals of the current work is to test whether the same mechanisms underlying semantic learning in neural network models-i.e., leveraging coherent covariation among features - might also underlie the emergence of structured category representations in humans.

\section{Embedding structure in models of category learning}

Whether and how structure influences initial category representations will have consequences for models of category learning. Prominent theories of category formation are not able to capture the kinds of feature-based structure that appear important for established concepts. The prototype theory, for instance, posits that a learned category representation reflects the central tendency of features across exemplars (Rosch, 1973; Rosch \& Mervis, 1975). Prototypes can explain the fuzziness of category boundaries (Hampton, 1995) and can predict a wide range of category judgments and categorization phenomena (Rosch \& Mervis, 1975; Rosch, 1973, cf. Medin et al., 1987). Models instantiating prototype theory have also had success in predicting human categorization performance (Smith \& Minda, 1998; 2002, Bowman et al., 2020; Devraj et al., 2021). Importantly, while a category prototype will reflect the feature frequencies across category members, it cannot capture the relations or associations between those features, including the coherent covariation that crucially contributes to neural network models' ability to account for real-world concept learning.

A contrasting view is offered by exemplar theory, in which categories are represented as a stored set of observed exemplars (e.g., Nosofsky, 2011). Whereas prototype theory argues that the features of individual exemplars are integrated into a single category representation, exemplar theory includes no representational integration across exemplars. Instead, exemplars are stored separately, and a subset of these exemplars are activated during category related judgments. Exemplar models have had significant success in predicting behavioral patterns of category learning (Medin \& Schaffer, 1978; Hintzman, 1984; Nosofsky, 1984; 1998; Nosofsky et al., 2018). Some neuroimaging evidence has found increased support for exemplar theory relative to prototype theory (Mack et al., 2013), though 
it has also been argued that both exemplar- and prototype-based representations coexist in the brain during category learning (Bowman et al., 2020). One possible explanation for the exemplar model's success is that meaningful variability across exemplars is maintained and this can benefit recognition and generalization (Nosofsky et al., 2018). Preserving the variability of features across exemplars adds a kind of structure to exemplar models that prototype models lack — the patterns with which features co-occur across category members is at least accessible across stored exemplars, even though these feature associations are not directly represented. It is unclear to what extent exemplar models can fully capture patterns of rich, structured learning, such as in the case of coherent covariation across features (Hinton, 1986).

Our hypothesis is that richer representations — relative to stored prototypes or individual exemplarsare required to process and learn structured categories. In particular, we believe that learning exemplars in distributed representations, in which populations of neurons are responsive to many related features of the environment, can lead to structured category representations containing meaningful feature associations. This kind of representation crucially contributes to the successful semantic development observed in neural network models, though (1) the structures learned by existing models are within and across larger semantic domains (e.g., ANIMALS, PLANTS) as opposed to within items (e.g., ROSE; c.f. McClelland \& Rumelhart, 1985), and (2) the semantic learning simulated in the models is presumed to occur over longer time scales (e.g., years of development in infants). Here we aim to extend the principles of these prior models to examine how the structure within an individual category is learned, and whether internal category structure influences how it is learned. By doing so, we will also be able to test how richer representations contribute to the rapid initial learning of novel category structure. According to complementary learning systems (CLS) theory, experiences are first encoded as sparse, non-overlapping representations in the hippocampus, and only during subsequent consolidation are they integrated into distributed representations in cortex (McClelland et al., 1995). However, our recent computational model of the hippocampus argues that distributed representations quickly emerge in subfield CA1, separate from the sparse representations of Dentate Gyrus and CA3 (Schapiro et al, 2017). We have also shown that the hippocampus is sensitive to overlapping associations and higher-level structure in the environment (Schapiro et al., 2016). This prior work leads us to predict that distributed representations may rapidly develop during initial encounters with a rich category structure. 
Our current approach will also enable us to test whether the same principles that govern learning of broad semantic domains also govern learning of individual items. If leveraging coherent covariation of semantic features facilitates the formation of representations of semantic categories, it seems likely that coherent covariation of features within a category might similarly benefit learning. In a category with clusters of reliably covarying features, it might be relatively easier to learn the features that are most important for that category, or there may be additional benefits such as improved category generalization (Bowman \& Zeithamova, 2021). We already know that humans are sensitive to the statistical structure found within many cognitive domains, as discussed below. An open question is whether humans are sensitive to feature-based category structure during initial learning, and further whether specific structures benefit or interfere with category learning and the building of useful category representations.

\section{Structure learning across cognitive domains}

Structure can manifest in many ways. It can be operationalized in terms of statistical co-occurrences, transitional probabilities, and physical proximities, among others. Some of the earliest empirical evidence that humans are sensitive to these kinds of structures was provided in the domain of language development. Saffran et al. (1996) reported that after brief exposure to a stream of nonsense syllables, infants were able to learn the pseudo-word boundaries based solely on the temporal contingencies between syllables. This statistical learning phenomenon has been reported across many domains (Frost et al., 2019).

Humans are also sensitive to higher-level structure that goes beyond pairwise statistics. Researchers often describe high-level temporal structure in terms of graphs, or networks (Karuza et al., 2016; Lynn \& Bassett, 2020). Graph nodes correspond to different stimuli, and the edges between nodes specify possible transitions between stimuli. Within an experiment, the topography of the graph is carefully designed in order to target elements of the learning process. A network topography that is often used in these experiments contains clusters of nodes, or "communities", such that the stimuli within a community tend to occur within close temporal proximity (Schapiro et al., 2013; 2016; Karuza et al., 2017; 2019; Kahn et al., 2018; Lynn et al., 2020; Mark et al., 2020; Kakaei et al., 2021; Pudhiyidath et al., 2020). The extent to which networks or graphs contain these communities is quantified in terms of "modularity" - networks with denser clusters of nodes exhibit higher modularity (Rubinov \& Sporns, 2011). Behavioral performance on statistical learning tasks reveals that humans are sensitive 
to community structure underlying visual (Schapiro et al., 2013; Kakaei et al., 2021), motor (Kahn et al., 2018), and navigational (Mark et al., 2020) tasks. It has been argued that modular structure leads to more accurate representations of a network (Lynn \& Bassett, 2020).

While structure learning has been witnessed across many cognitive domains-including language, visual perception, motor actions, and navigation-structure learning paradigms have not yet been applied to novel category learning. When a category is modeled as a graph containing features and feature-associations (Solomon et al., 2019), modular structure describes coherent covariation across features. That is, a modular "community" would, in this case, reflect a set of features that tend to cooccur with high probability across exemplars. In this way, we can compare carefully designed graph structures to assess how coherent covariation within a category influences learning. This approach will reveal aspects of category and concept learning specifically, but also will inform theories and models of structure learning more broadly.

\section{Overview of experiments}

We aimed to synthesize structure learning and category learning paradigms to examine how structured categories may give rise to structured representations. Evidence that structured category representations are rapidly built during learning would forge a link between novel category representations and the rich representations known to underlie established concepts. In a series of behavioral experiments, human participants learned novel animal categories whose feature association statistics were determined by carefully manipulated underlying graph structures. We specifically compared a Modular graph structure like the ones described above-containing clusters of covarying features - with two non-modular structures (i.e., Random, Lattice). The specific features assigned to the graph nodes were randomized for each participant in order to isolate the effects of the category structure. We ran simulations to test whether a neural network model can replicate patterns of human category learning, and compared the results to standard exemplar and prototype models.

We predicted that humans would be able to learn the feature-based category structures, based on successful performance on prior statistical learning tasks. More importantly, we predicted that the specific structure of a category (i.e., modular vs. non-modular) would influence the ease with which that category is learned. If the mechanisms involved in learning an individual category parallel those involved in semantic learning more broadly, we would expect increased performance on Modular 
categories, relative to Random and Lattice categories, due to the coherent covariation between features in the modular graph communities. We predicted the same for our model, as neural network models employing distributed representations should be especially attuned to this coherent covariation (Rogers \& McClelland, 2004).

We tested these predictions across three experiments, examining human behavior and model simulations. In Experiment 1, we designed two novel animal categories defined by lists of verbal features whose co-occurrence statistics were dictated by a Modular or Random graph structure. Since our empirical questions relate to internal category structure as opposed to category distinctions, we exposed participants to the two categories in a missing feature task, an inferential task that promotes within category comparisons (Yamauchi \& Markman, 1998; Anderson et al., 2002; Chin-Parker \& Ross, 2002; 2004; Markman \& Ross, 2003; Erikson et al., 2005). To preview our results, we found support for the prediction that feature learning was improved in the Modular relative to the Random category; our neural network model accounted for this effect, whereas exemplar and prototype models could not. In Experiment 2, we replicated the results of Experiment 1 using novel visual insect stimuli to show that this effect replicates across stimulus modality. In Experiment 3 we compared Modular and Lattice category learning using our novel insects in a statistical learning paradigm to test whether our category structure effects emerge even when structure is incidental to the learning task. We again found evidence that a Modular category structure improved category learning, and that only the neural network model could account for these results. In sum, these experiments suggest that category learning is influenced by its underlying feature-based structure, and that the resulting new concepts likely take the form of rich, distributed representations.

\section{Experiment 1}

In Experiment 1, human participants were exposed to two novel animal categories whose features were presented in a verbal format. Participants learned the features of these categories in an inferential missing features task, in which a set of features (i.e., an "exemplar") was presented and participants selected the feature they believed was missing out of three possible options. Unbeknownst to the participants, the internal structure (i.e., pattern of feature correlations) differed between the two categories: the Random category only contained one cluster of "core" features and no peripheral 
structure (Fig. 1B), whereas the Modular category consisted of an identical core structure in addition to a peripheral structure with two clusters of correlated features (Fig. 1A). This experiment allows us to evaluate whether the internal structure of a category influences how that category is learned.

An advantage for the Modular category would suggest that people are sensitive to the coherent covariation of features created by the modules. This learning pattern would be consistent with neural network models of semantic learning revealing that coherent variation among features benefits learning in broader semantic domains (Rogers \& McClelland, 2004). We thus ran simulations of a neural network model, exemplar model, and prototype model to determine whether the distributed representations that emerge in a neural network model can uniquely explain patterns of human category learning.

\section{Exp. 1 Behavioral Methods}

Participants. Forty (40) participants recruited from Amazon Mechanical Turk contributed data to Experiment 1 (Age: $M=37.5, S D=11.3 ; 64 \%$ female) and were compensated $\$ 4.50$ for their time. An additional five participants did not pass attentional checks (see catch trials below) and were excluded from analyses. Consent was obtained for all participants in accordance with the University of Pennsylvania IRB.

Categories and features. Two "species" of novel animals were created, each defined by 11 features that were presented in verbal form. One species contained the features large, two legs, solitary, blue eyes, bushy tail, sleeps in caves, has horns, growls, brown fur, drinks water, and striped. The other species contained the features small, four legs, social, grey eyes, hairless tail, sleeps in trees, has claws, roars, black fur, drinks milk, and spotted. The dimensions specified by the 11 features in each species were approximately matched (e.g., size, eye color, markings). In order to add additional variability to the category exemplars, for each species we generated an additional 40 features of the form "eats - " (e.g., eats lemons, eats lilacs, eats corn). We also chose an additional 6 features per species to use as catch features during the behavioral task (e.g., has fangs, white feet). No features overlapped between species. All features used in Exp. 1 are shown in Supplementary Table 1. Assignment of species to category label (i.e., Timbo, Sudex) was randomized for each participant. 
Category structures. Experiments 1 and 2 compared Random and Modular structures (Fig. 1). Both graphs contained the same core structure: three high-frequency features that are found in all exemplars. However, the peripheral structures of the graphs differed: in the Modular structure, the peripheral features were divided into two modules, or clusters, such that features from one cluster never co-occurred with features from the other cluster within the same exemplar. In the Random structure, every peripheral feature could co-occur with any other peripheral feature within an exemplar. Note that our Random structure is not named to reflect a "random network" topology (Watts \& Strogatz, 1998), but rather the fact that exemplars can have any random combination of peripheral features. Assignment of species to structure was randomized across participants. The features assigned to the core nodes were fixed within species (i.e., large, two legs, and solitary were always the core features for one category and small, four legs, and social were always the core features for the other category), but the assignment of features to the peripheral nodes was randomized. As a result, the features corresponding to each of the clusters in the Modular category differed across participants.

Category exemplars. Each exemplar was originally defined by six features, the combination of which was determined by the underlying graph structure (i.e., three core, three peripheral). Within these parameters, the Modular graph generates eight unique exemplars $\left({ }_{4} \mathrm{C}_{3} \times 2\right.$ modules) and the Random graph generates 56 unique exemplars $\left.{ }_{8} \mathrm{C}_{3}\right)$. To reach the 72 total exemplars per category needed for the behavioral task, we used 9 sets of the 8 unique exemplars from the Modular category; for the Random category, we used the 56 unique exemplars and subsampled an additional 16 from the same set to reach a total of 72 exemplars. Each exemplar was also assigned an idiosyncratic eats feature; each eats feature was seen in only one or two exemplars throughout the experiment. Thus, each exemplar contained a total of seven features: three core features, three peripheral features, and one idiosyncratic feature.

Task design. Each of the 72 exemplars from each category corresponded to a trial in the behavioral task. On each trial, one of the exemplar's features was removed and three possible features were presented as response options. The identity of the missing features and the identity of the three feature options was determined by one of four task conditions: category boundary, core structure (Random and Modular), and peripheral structure (Modular). In category boundary trials, a Random category exemplar was presented with a peripheral feature missing; the three options included the correct 
A

\section{MODULAR}
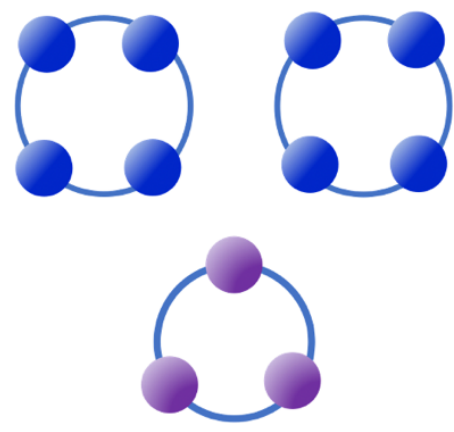

B

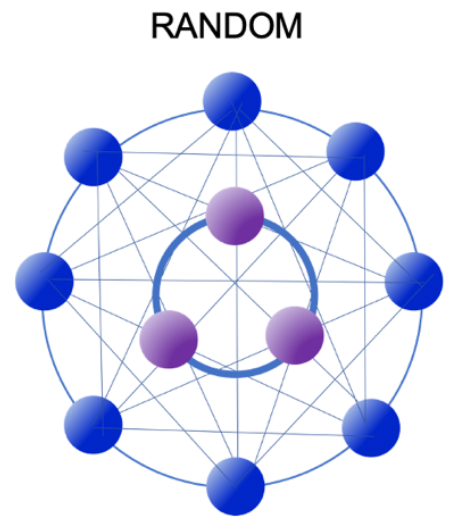

C

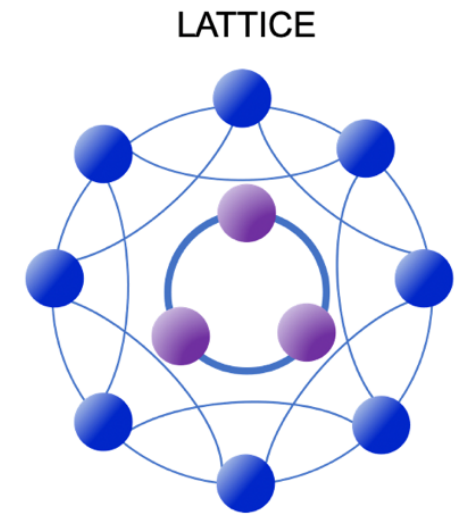

Figure 1: Category structures used across the experiments. Nodes indicate features and edges indicate their co-occurrence statistics. All graphs contained the same core structure, corresponding to three high-frequency features (purple nodes) sharing strong co-occurrence statistics (indicated by the thick edges). All core nodes share an edge with all peripheral nodes but these are not visualized for simplicity. (A) The Modular structure contained two clusters of peripheral features such that features in one module could never co-occur with features in the other module. (B) The Random structure contained peripheral features (blue nodes) with no clustering; any peripheral feature could co-occur with any other peripheral feature. (C) The Lattice structure, like the Random structure, contained no clustering in its periphery, but each peripheral feature could only cooccur with a subset of all peripheral features.

peripheral feature from the Random category and two Modular category peripheral features. These trials tested participants' ability to learn which features defined each category. To test core structure in both Random and Modular categories, an exemplar was presented with a core feature missing; the three options included the correct core feature and two peripheral features from that category. These trials tested participants' ability to learn that the three core features were necessary for each exemplar. (Note that in the Modular category, because each module contained only four features, one of the two incorrect features was from an exemplar-inconsistent module.) To test peripheral structure in the Modular category, an exemplar was presented with a peripheral feature missing (e.g., from module1); the three options included the correct module1 feature and two incorrect features from module2. These trials tested participants' ability to learn that features from separate modules could not co-occur. The 144 experimental trials were evenly divided between the boundary, core, and peripheral conditions: 48 category boundary trials (Random), 48 core structure trials (24 Random, 24 Modular), and 48 peripheral structure trials (Modular). An additional six catch trials (3 Random, 3 Modular) were designed in which a peripheral feature was removed and the options included the correct feature in addition to two features never before seen by the participant. Five participants who responded 

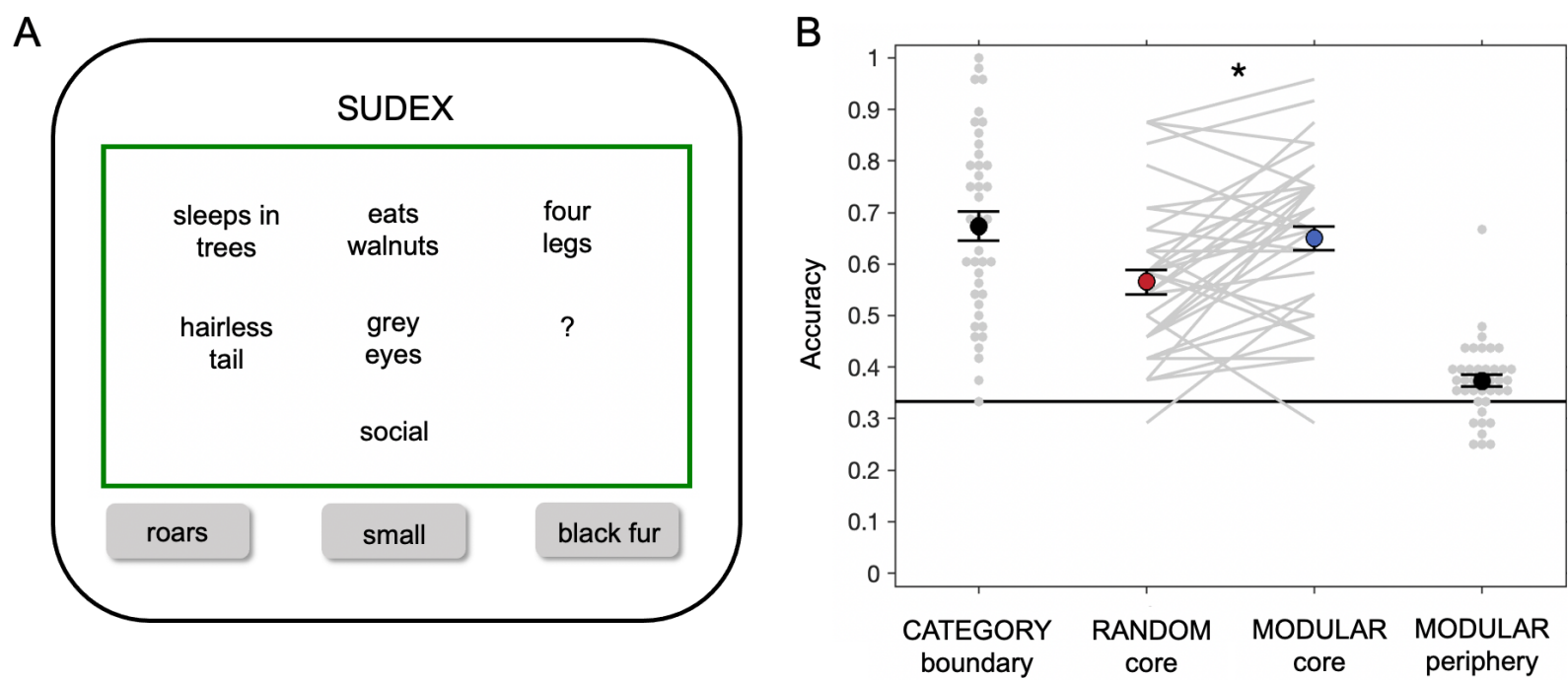

Figure 2: Experiment 1 design and results. (A) On each trial of the missing feature task in Exp. 1, the relevant category label was displayed (e.g., "sudex", "timbo") above a box containing an exemplar's features. Five structure-consistent features were displayed in addition to an idiosyncratic "eats" feature. A missing feature was indicated by a question mark and participants decided which of three possible features belonged to the exemplar. (B) Human participants successfully learned the category boundary, Random core structure, Modular core structure, and Modular periphery structure. Accuracy was significantly higher for Modular vs. Random core structure $(t(39)=3.8, p=0.0004)$, even though core structure was identical across categories. Error bars reflect standard error of the mean.

incorrectly to 3 or more of the 6 catch trials were excluded from analyses. Trial order was pseudorandomized such that exemplars from a single category were seen approximately 5 times in a row before switching to the other category, and all catch trials occurred in the second half of the experiment.

The frequencies with which features appeared on exemplars were balanced within each structure condition: each core and peripheral feature in the Modular category appeared in 64 and 21 exemplars, respectively; these frequencies were identical in the Random category. Core and peripheral features appeared as the correct response option on 8 and 6 trials, respectively, in both structure conditions. However, the category boundary condition made it impossible to balance the extent to which features appeared as an incorrect response: each Random peripheral feature appeared as an incorrect response on 6 trials, whereas each Modular peripheral feature appeared as an incorrect response between 27-33 times. Core features were never presented as incorrect response options.

Verbal Missing Feature Task. Participants completed 150 trials of the missing feature task. Before the task began, the participants were told: "We will train you on the animal categories by showing you 
category members with one feature missing. On each trial, you will be given three features and you will choose the feature that you think is the missing one." On each trial, an exemplar's features were presented inside a box in the center of the screen, and three feature options were presented beneath the exemplar (Fig. 2A). The order of the exemplar's features inside the box, and the order of the three response options, was randomized on each trial. Participants could take as long as they needed to select a feature by clicking the corresponding button. When a participant made a correct response, their selection would be outlined in green and they would proceed to the next trial. When a participant made an incorrect response, their selection would be outlined in red and the trial was repeated until the correct feature was chosen. The positions of exemplar features and response options were randomized each time a trial was repeated. Feedback was presented immediately after a feature option was clicked and remained on screen for 1000 ms; the subsequent trial began 1500 ms after the response was made.

Statistical Analysis. Each trial was coded as accurate (1) or inaccurate (0) based on the first response given (i.e., a trial was similarly marked as incorrect whether it took two or more than two times to choose the correct feature). Mean accuracies for category boundary, Random core structure, Modular core structure, and Modular peripheral structure trials were calculated for each participant. Accuracy across participants was compared to chance (0.33), and core structure knowledge in Random and Modular categories was compared using a paired t-test. Relationships between different kinds of category knowledge were assessed using Pearson correlations.

\section{Exp. 1 Behavioral Results}

Accuracy for verbal missing features. Mean accuracies across participants for the four structure conditions are shown in Fig. 2B. Above chance accuracy was observed in the category boundary $(M=67.4 \%$; $S D=18 \% ; t(39)=11.9, p<0.0001)$, Random core $(M=56.6 \% ; S D=15.2 \% ; t(39)=9.7 \mathrm{p}<0.0001)$, Modular core $(M=65.0 \% ; S D=14.8 \% ; t(39)=13.5, p<0.0001)$, and Modular periphery $(M=37.3 \%$; $S D=7.4 \% ; t(39)=3.4, p=0.002)$ conditions. A one-way ANOVA revealed a significant difference between conditions $(F(3)=35.9, p<0.0001)$. Pairwise dependent t-tests revealed that accuracy for Modular periphery structure was significantly lower than all other kinds of structure knowledge: category boundary $(t(39)=10.3, p<0.0001)$, Random core $(t(39)=7.9, p<0.0001)$, and Modular core 
$(t(39)=11.5, p<0.0001)$. Category boundary accuracy exceeded Random core accuracy $(t(39)=3.3$, $p=0.002)$ but was not significantly higher than Modular core accuracy ( $p>0.4)$. Most interestingly, Modular core accuracy was significantly higher than Random core accuracy $(t(39)=3.8, p=0.0004)$, despite the fact that core structure was identical across categories.

Relationships between kinds of structure knowledge. Accuracy for the Random core and Modular core conditions was significantly correlated across participants $(r(39)=0.57, p=0.0001)$. No relationship between Modular core and periphery accuracy was observed ( $p>0.2)$. Category boundary accuracy did not reliably predict Random $(r(39)=0.25, p=0.13)$ or Modular $(r(39)=0.30, p=0.06)$ core accuracy; it notably also did not predict the increase in accuracy for Modular core vs. Random core structure $(r(39)=0.05, p=0.74)$. This suggests that the observed Modular core benefit does not emerge merely because participants learned to avoid Modular-peripheral features on category boundary trials. Similarly, Modular periphery accuracy did not predict the Modular core structure benefit $(p>0.9)$, further suggesting that higher accuracy on Modular core trials is unlikely to be due to participants' ability to exclude peripheral features from the inconsistent module on Modular core trials.

\section{Exp. 1 Modeling Methods}

\section{Neural Network Model}

Model architecture and parameters. We constructed our model in the Emergent simulation environment (O'Reilly et al., 2020). A schematic of our model architecture is shown in Fig. 3A. The model comprised an input layer (22 units), one hidden layer (400 units), and an output layer (22 units). The units in the input and output layers corresponded to the 22 total features across the two categories. The input layer had full feedforward connectivity to the hidden layer, and there was full bidirectional connectivity between the hidden layer and output layer. We used Emergent's default parameters and training regime, which implements the eXtended Contrastive Attractor Learning (XCAL) rule. We expect, however, that any error driven learning rule (e.g., backpropagation) operating on distributed representations would behave similarly in our settings.

Training protocol. The model was trained as an autoencoder on full exemplars that corresponded with the 144 experimental trials used in the behavioral experiment (i.e., the 5 shown features plus the 1 
correct feature). That is, training consisted of 144 trials in which the model was presented with a sixfeature exemplar on the input layer and learned to recreate the six-feature exemplar on the output layer. The order of training trials was randomized.

Testing protocol. Test trials were analogous to the experimental trials in the missing feature task. A total of 144 trials tested category boundary knowledge (48), Random core structure knowledge (24), Modular core structure knowledge (24), and Modular peripheral structure knowledge (48). On each trial, five features were presented on the input layer (i.e., an exemplar with a missing feature) and we analyzed activity in the output layer to determine whether the model was able to successfully activate the correct feature. We restricted this analysis to the same three features that were presented to human participants; a test trial was coded as accurate (1) if the model activated the correct feature more strongly than the two incorrect features, and incorrect (0) if one of the incorrect features was the most strongly activated unit. The full set of test trials (144) was run after every 8 training trials for a total of 18 test epochs per simulation.

Model assessment. We ran the model 100 times, with a new initialization of weights and randomized trial order for each simulation. Within each simulation, the mean accuracy for each of the four conditions was calculated within each test epoch. We calculated mean accuracy across test epochs for category boundary, Random core, Modular core, and Modular peripheral conditions for each simulation. Accuracy was compared to chance (0.33) and paired t-tests were used to compare Random and Modular core structure knowledge.

Analyzing feature representations. In order to assess how feature representations in the model transformed during learning, we recorded the pattern of activity evoked across the hidden layer units on test trials where each feature was presented by itself. Thus, after every eight training trials we obtained patterns of activity representing the 22 category features. For each simulation, we simultaneously ran multidimensional scaling on the 18 test epochs $\times 22$ feature representations using Euclidean distance, enabling us to visualize how the features became more or less similar to each other across learning (Fig. 3C). Because of the simultaneous calculation of the distances across all time points, the initial starting points of the 22 features appear to be meaningfully differentiated but are are not. 
A

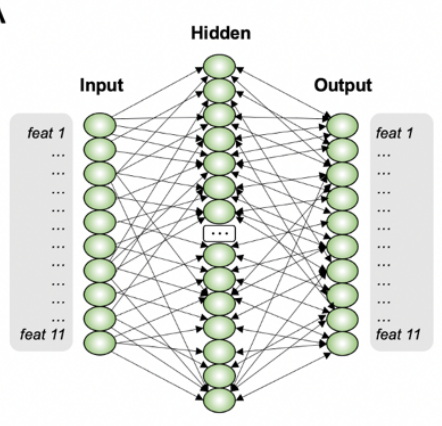

B

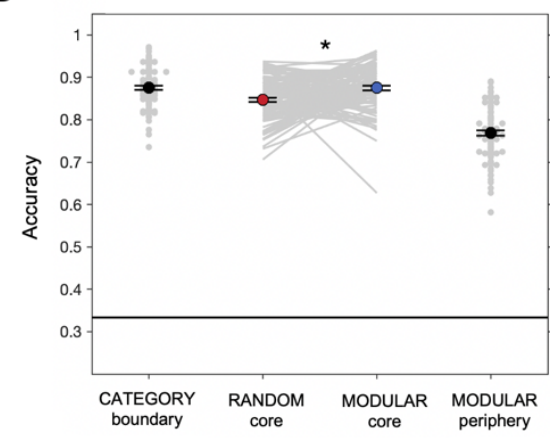

C

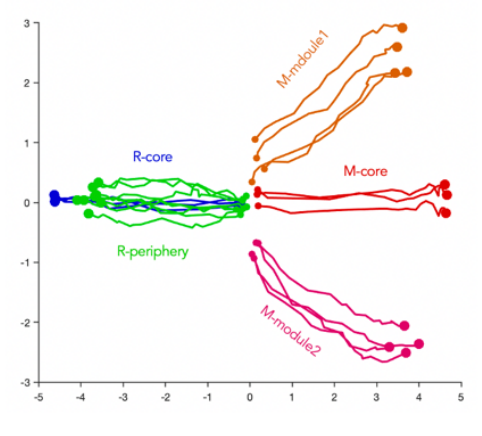

Figure 3: Neural network model simulation of the missing feature task. (A) The model architecture contained 22 feature nodes on the input and output layers and a 400-unit hidden layer. (B) Across 100 simulations, the model replicated patterns of human performance such that higher accuracy was observed in the Modular core vs. Random core conditions $(t(99)=3.5, p=0.0006)$. Error bars reflect standard error of the mean. (C) Multidimensional scaling solution revealing how feature representations change from the beginning (center small dots) to the end (large dots) of training for Random (cool colors) and Modular (warm colors) categories. Modular core (red) and peripheral (orange, pink) features are more strongly differentiated by the end of learning relative to the Random core (blue) and peripheral (green) features.

\section{Exemplar Model}

Adapting the generalized context model (GCM). We adapted the exemplar model equations from Nosofsky et al. (2018) to our current design. The original GCM is concerned with quantifying the probability that an exemplar is classified into one of two or more categories. Here we do not focus on category boundaries but rather internal category structure; we therefore simplified the equation to reflect the match of a current test stimulus to its corresponding category. The probability (or the match value) of category $J$ given test stimulus $i$ was calculated as the summed similarity of stimulus $i$ to all previously observed exemplars $j$ in category $J$ :

$$
P\left(C_{J} \mid i\right)=\sum_{j \in J} s_{i j}
$$



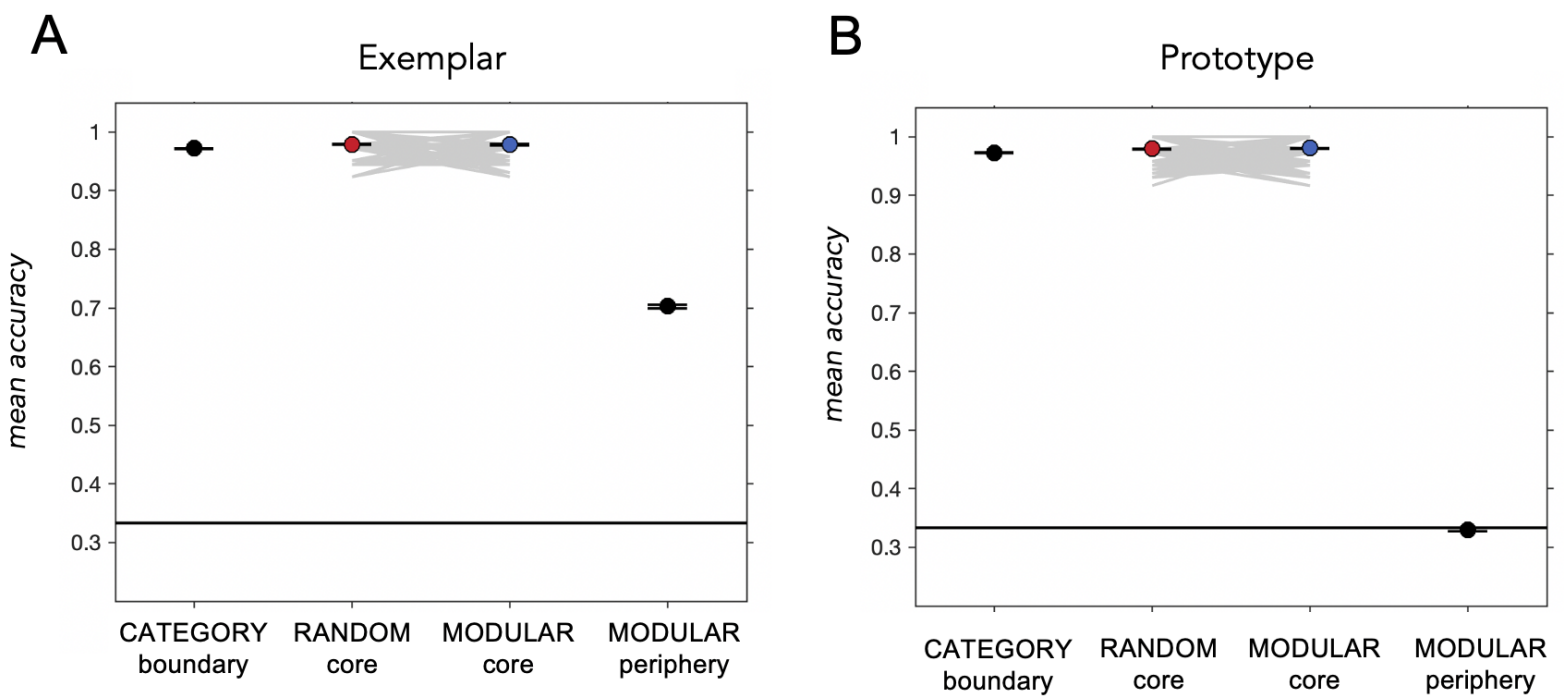

Figure 4: Exemplar and prototype model simulations of the missing feature task. (A) The exemplar model performed above chance in all structure conditions, but revealed no difference between Random and Modular core structure learning $(p>0.9)$. (B) The prototype model also performed similarly on Random and Modular core structure trials $(p=0.37)$; in contrast with the exemplar model, the prototype model could not learn Modular periphery structure. Error bars reflect standard error of the mean.

where $i$ and $j$ are binary vectors with 11 values representing the presence (1) or absence (0) of each of the 11 category features, and $s$ represents the similarity of the current stimulus $i$ to each stored exemplar $j$. This similarity measure is an exponential decay function of the distance between $i$ and $j$ in multidimensional feature space:

$$
s_{i j}=\exp \left(-c \cdot d_{i j}\right)
$$

The sensitivity parameter $c$ determines the rate at which similarity declines with distance; any positive value of $c$ means that exemplars that are in close proximity with the test stimulus will have the greatest influence on its match value. Nosofsky et al. (2018) reported an optimized sensitivity parameter value of $\sim 1.8$; we thus tested this parameter value specifically in addition to a wider range of values. Since our category features were represented as binary values, $d$ reflects the Hamming distance between items. These equations were used to calculate the match of each test stimulus to its corresponding category in the analyses described below.

Model assessment. In the missing feature task, humans were given feedback on each trial such that by the end of each trial they could encode a correct 6-feature exemplar (the five probed features plus the 
correct missing feature). In our exemplar model simulations, we therefore considered the stored exemplars of category $J$ to be the learned 6 -feature exemplars of category $J$ from all previous trials. On each trial, three test stimuli were compared with the stored exemplars, corresponding to the three missing feature options for that trial. The correct stimulus $i_{0}$ contained six features: the five probe features plus the correct missing feature. Each of the two incorrect exemplars, $i_{1}$ and $i_{2}$, contained the five probe features plus one of the incorrect features. We calculated $P_{0}, P_{1}$, and $P_{2}$ as described above, where category $J$ is always the correct category (if the current correct test stimulus is from a Modular category, we only compare that stimulus with previously seen Modular exemplars). That is, the match value of correct stimulus $i_{0}$ on trial $t$ is calculated as the summed similarity of $i_{0}$ with all $j$ exemplars from trial 1 to $t-1$. We calculated the match of incorrect stimuli $i_{1}$ and $i_{2}$ in the same manner, and compared match values to determine the accuracy on each trial. If $P_{0}$ was greater than both $P_{1}$ and $P_{2}, a c c=1$; if $P_{0}$ was equal to the greater value amongst unequal $P_{1}$ and $P_{2}, a c c=1 / 2$; if all values were equal, $a c c=1 / 3$; and if either $P_{1}$ or $P_{2}$ was greater than $P_{0}, a c c=0$. Mean accuracy was calculated separately for Random core and Modular core trials. Within each of 500 model simulations, trial order was randomized and mean accuracy was calculated within each condition.

\section{Prototype Model}

The prototype model was nearly identical to the exemplar model, except instead of comparing the test stimulus to each observed exemplar $j$, it was compared to the centroid of all previously seen exemplars in category $J$. The centroid was represented as the mean across exemplars, resulting in a vector with 11 feature values between 0 and 1. Because this prototype vector was not binary, we used Euclidean distance to calculate the stimulus match values in the prototype model (Nosofsky et al., 2018).

\section{Exp. 1 Modeling Results}

Neural network model. Mean model accuracy across simulations is shown in Fig. 3B. As with human participants, the model was able to learn the category boundary $(\mathrm{M}=87.5 \%, \mathrm{SD}=4.7 \%)$, Random core $(\mathrm{M}=84.7 \%, \mathrm{SD}=5.2 \%)$, Modular core $(\mathrm{M}=87.5 \%, \mathrm{SD}=5.3 \%)$, and Modular periphery $(\mathrm{M}=76.9 \%$, $\mathrm{SD}=6.1 \%)$ at above chance levels $\left(p^{\prime} \mathbf{s}<0.0001\right)$. Most interestingly, the model replicated the Modular 
core benefit observed in human participants: accuracy was significantly greater for Modular core structure than for Random core structure $(t(99)=3.5, p=0.0006)$. Visualization of the training course MDS solution reveals that the model learns to more strongly differentiate the core from peripheral features in the Modular categories relative to the Random categories (Fig. 3C).

Exemplar model. Mean exemplar model accuracy is shown in Fig. 4A. For simplicity, we compare accuracy across the four structure conditions when sensitivity parameter $c=1.8$. The exemplar model successfully learned the category boundary $(M=97.2 \%, p<0.0001)$, and also performed above chance on Random core $(M=97.9 \%, p<0.0001)$ and Modular core $(M=97.9 \%, p<0.0001)$ trials. However, we observed no difference between Random and Modular core trials $(t(499)=0.044, p>0.9)$. The exemplar model also successfully learned the Modular peripheral structure $(M=70.3 \%, p<0.0001)$. A nearly identical pattern of results was observed across a range of sensitivity parameter values: in no case did the exemplar model predict increased accuracy for Modular core relative to Random core trials (Supplementary Fig. 1A).

Prototype model. The prototype model performed similarly to the exemplar model (Fig. 4B). It successfully discriminated between categories $(M=97.3 \%, p<0.001)$, learned Random core $(M=97.9 \%$, $p<0.0001)$ and Modular core $(M=98.1 \%, p<0.0001)$ structure successfully, yet exhibited no difference between Random and Modular core structure $(t(499)=0.91, p=0.37)$. However, in contrast with the exemplar model, the prototype model was not able to learn Modular peripheral structure $(M=33.0 \%$, $p=0.29)$.

\section{Exp. 1 Discussion}

In Experiment 1, we found that human participants were able to learn the rich internal structures of two novel categories. Most notably, participants found it easier to learn the core features of the Modular category relative to the Random category, even though core structure was identical across categories. This suggests that the clustered feature structure benefitted overall understanding of the Modular category. The same pattern of results arose in our neural network model of the task, but not in exemplar or prototype models. Analyses of the learned internal representations in the neural network model revealed that the representations of the peripheral features pulled apart from the core 
features much more so in the Modular category, as the network was highly sensitive to the feature correlations within the modules. This sensitivity appears to require rich internal representations; classic exemplar and prototype models did not exhibit any difference in sensitivity to the core features across categories.

The results are consistent with prior demonstrations that neural network models with distributed representations are highly attuned to coherent covariation among features at larger semantic scales (Rogers \& McClelland, 2004). Learning the structure of large semantic domains (e.g., ANIMALS) and learning the internal structure of individual concepts (e.g., FOX) might involve similar mechanisms of extracting the correlational structure of features across items or exemplars (McClelland \& Rumelhart, 1985). Just as the clustering of reliably co-occurring features provides traction on learning the boundaries between concepts, as has been previously shown, clusters of reliably co-occurring features within a concept aid the formation of a learned, structured category representation that can be used to generalize across category members and support feature inference. Both humans and the neural network model learned this new structure rapidly, orders of magnitude more rapidly than in the learning of large semantic domains. This suggests that neither humans nor neural network models require large amounts of training to build rich distributed representations.

The model's representation of the task was abstract and not tied to the particular verbal nature of the stimuli, suggesting that the Modular core benefit observed in Experiment 1 should manifest in other domains as well. We thus predicted that we would observe a similar effect when categories are presented not as lists of features but as visual objects.

\section{Experiment 2}

The results of Experiment 1 suggest that it is easier for humans to learn the core structure of a Modular category relative to a Random category, indicating a sensitivity to coherent covariation among the clustered features. Experiment 2 aimed to replicate this effect using visually- rather than verballypresented categories, based on our expectation that category structure learning effects emerge from a 
domain-general learning mechanism. Experiment 2 was thus designed to test whether the Modular core benefit is robust across feature types.

\section{Exp. 2 Methods}

Participants. Forty (40) participants recruited from Amazon Mechanical Turk contributed data to Experiment 2 (Age: $M=40.6, S D=12.0 ; 53 \%$ female) and were compensated $\$ 4.50$ for their time. An additional eight participants did not pass the attentional checks and were excluded from analyses. Consent was obtained for all participants in accordance with the University of Pennsylvania IRB.

Categories and features. Two species of novel insects were created with 11 features each, now displayed in visual form ("beetle" and "butterfly"; Fig. 5AB). Each species consisted of an insect base on which 11 various animal features could be attached. The general kind and location of features were matched across the species: horns/antennae (3), sets of wings (2), sets of arms (2), colored markings (2), tail (1), and toe features (1). These stimuli were designed such that any subset of the 11 features could be added to the base to form a potential category exemplar. An additional six features were generated for each species to use in the catch trials. The category base and features were created in Adobe Illustrator by cropping and editing open-source images of real animals found on the internet. No category labels were used in Experiment 2.

Category structures and exemplars. The same Random and Modular structures used in Experiment 1 were used in Experiment 2. Assignment of species to structure was randomized across participants, and assignment of features to graph nodes was fully randomized for each participant (i.e., any feature could be a core feature). The set of visual exemplars in Experiment 2 were generated using an identical procedure to the one used in Experiment 1, resulting in 72 6-feature exemplars within both the Random and Modular categories. Instead of assigning an idiosyncratic feature to the visual exemplars, the color of the insect base was randomly adjusted slightly on each trial to add additional variability across exemplars. 
Task design. The design of Experiment 2 was identical to Experiment 1, resulting in a total of 144 experimental trials and 6 catch trials. Participants who didn't respond correctly to at least 4 of the 6 catch trials $(N=8)$ were excluded from subsequent analyses.

A

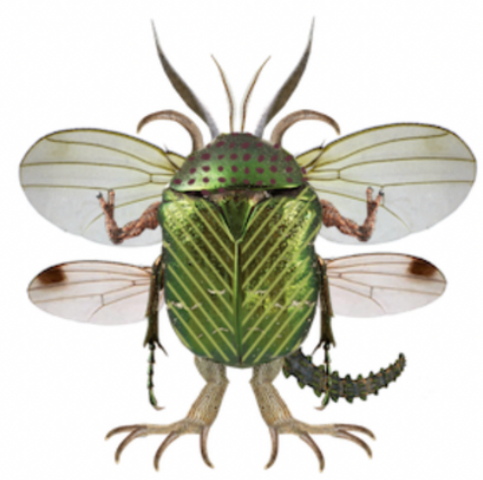

C

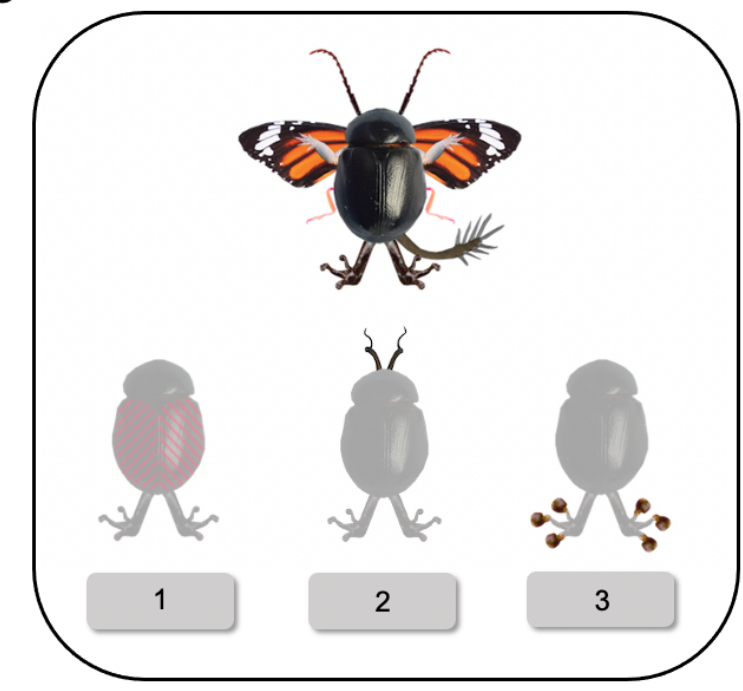

B

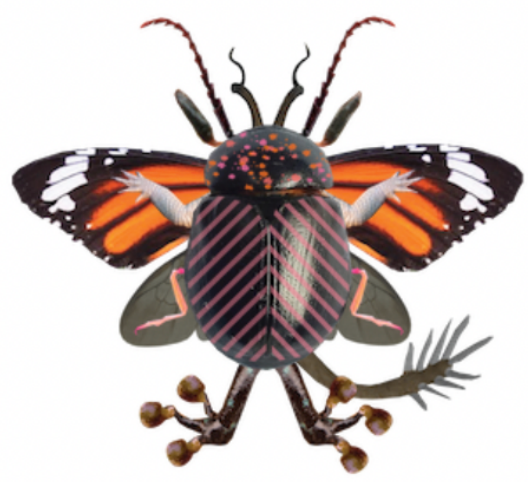

D

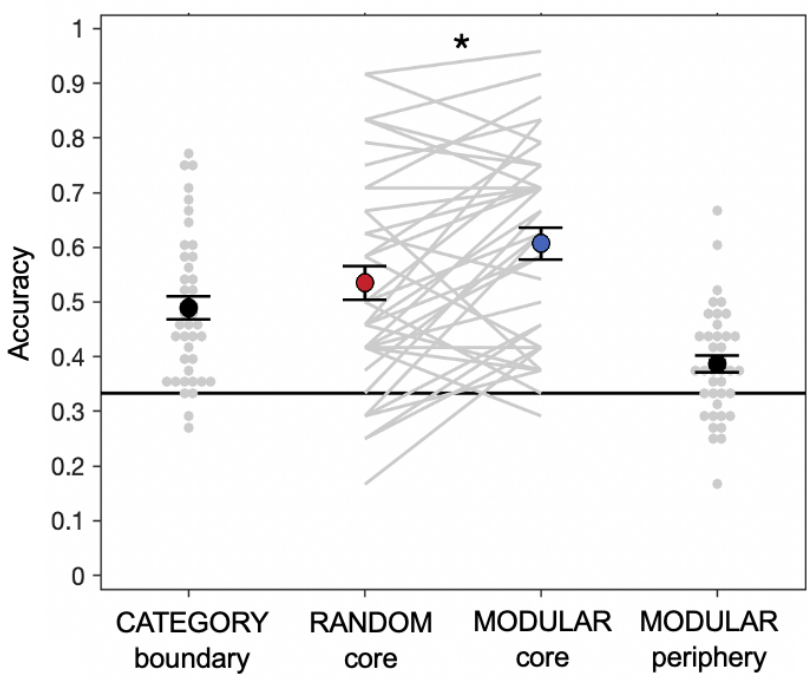

Figure 5: Visual missing feature task in Experiment 2. The (A) "beetle" and (B) "butterfly" species each consisted of an insect base upon which 11 features could be presented. (C) On each trial, an exemplar with five features was presented and participants selected which of three possible features they thought belonged. (D) The Modular core benefit was replicated in Experiment 2 such that participants' accuracy was significantly higher for the Modular core vs. Random core trials $(t(39)=2.8, p=0.007)$.

Visual Missing Feature Task. Participants completed 150 trials of the missing feature task, and were given the same instructions used in Experiment 1. On each trial, the visual exemplar with five features was presented in the center of the screen, and the three feature options were displayed below (Fig. 3C). The feature options were presented on a desaturated insect base above a numbered button (on 
category boundary trials, the insect base consistent with the displayed exemplar was used even though it displayed a feature from the incorrect category). Participants could take as long as they needed to click on the button corresponding to their feature choice. Feedback was presented in an identical way to Experiment 1 and trials were repeated until the correct feature choice was made.

Statistical Analysis. Mean accuracy for each participant and each condition was calculated as in Experiment 1. Accuracy for each condition was compared to chance (0.33), and conditions were compared with paired t-tests and Pearson correlations.

\section{Exp. 2 Results}

Accuracy for visual missing features. Mean accuracies across participants for the four structure conditions are shown in Fig. 3D. As in Experiment 1, above chance accuracy was observed in the category boundary $(M=48.9 \% ; S D=13.5 \% ; t(39)=7.3, p<0.0001)$, Random core $(M=53.5 \% ; S D=19.4 \%$; $t(39)=6.6, p<0.0001)$, Modular core $(M=60.7 \% ; S D=18.1 \% ; t(39)=9.6, p<0.0001)$, and Modular periphery $(M=38.7 \% ; S D=9.9 \% ; \quad t(39=3.4, p=0.002)$ conditions. A one-way ANOVA revealed a significant difference between conditions $(F(3)=13.9, p<0.0001)$. Pairwise dependent t-tests revealed that accuracy for Modular periphery structure was significantly lower than all other kinds of structure knowledge: category boundary $(t(39)=4.2, p<0.001)$, Random core $(t(39)=5.0, p<0.001)$, and Modular core $(t(39)=7.7, p<0.001)$. Category boundary accuracy did not significantly differ from Random core accuracy $(t(39)=1.5, p=0.15)$ but was significantly lower than Modular core accuracy $(t(39)=3.7$, $p<0.001)$. Most importantly, we replicated the Modular core benefit using visual categories: Modular core accuracy was significantly higher than Random core accuracy $(t(39)=2.8, p=0.007)$, despite the fact that core structure was identical across categories.

Relationships between kinds of structure knowledge. Accuracy for Random core and Modular core structures was significantly correlated $(r(39)=0.63, p<0.0001)$. No significant relationship between Modular core and periphery accuracy was observed $(r(39)=0.26, p=0.10)$. Category boundary accuracy did not reliably predict Random $(r(39)=0.31, p=0.052)$ or Modular $(r(39)=0.19, p=0.23)$ accuracy; it notably also did not predict the increase in accuracy for Modular core vs. Random core structure $(r(39)=-0.16$, 
$p>0.3)$. Similarly, Modular periphery accuracy did not predict the Modular core structure benefit $(r(39)=-0.10, p>0.5)$.

\section{Exp. 2 Discussion}

Using visual category stimuli, Experiment 2 replicated the behavioral Modular core benefit observed in Experiment 1. Participants were exposed to two novel insect categories defined by sets of discrete, non-overlapping features in a missing feature task. Even though core structure was identical across the Modular and Random categories, participants found it easier to learn the three core features of the Modular category. These results further support the claim that humans leverage feature-based statistics across category members to learn structured category representations, and that these representations support successful feature inference. The replication across stimulus types suggests that a domaingeneral learning mechanism is at play.

While results from Experiments 1 and 2 converge on the finding that increased clustering benefits the learning of stable category features, there are two possible alternative explanations. The first relates to the distribution of features in the missing feature task. In the displayed exemplars, feature frequencies were exactly balanced - core features appeared with equal frequencies in the Random and Modular categories, as did peripheral features. The frequency with which core and peripheral features appeared as the correct feature response was similarly balanced across category structures. However, the feature frequencies in the set of incorrect feature responses were not balanced-Modular peripheral features appeared more frequently as incorrect options than did Random peripheral features. It is possible that participants learned to reject Modular peripheral features in the missing feature task, thereby increasing the likelihood that they would respond correctly on the Modular core trials. This cannot explain the Modular benefit observed in the model simulations, and our correlational analyses on human behavior do not lend support to this explanation. However, we aimed to design another experiment that would eliminate this possibility entirely.

The second possibility is that the differences observed between the Modular and Random categories is not due to modularity or clustering, per se, but to some other graph property that differs across the 
two structures. While it is true that the Modular graph contains two distinct peripheral clusters while the Random graph does not, it is also true that there is less connectivity between Modular peripheral features overall—specifically, each peripheral node in the Modular graph is connected to three other peripheral nodes, whereas each Random peripheral node is connected to seven other peripheral nodes. We thus aimed to design a new graph structure that still contained no peripheral clustering but was better matched with the Modular graph in terms of peripheral node connectivity. The presence of peripheral structure in this new graph would additionally enable a more balanced task design and would help remove the constraints that contributed to the feature frequency imbalance in Experiments 1 and 2.

\section{Experiment 3}

In Experiments 1 and 2, humans and models were sensitive to the coherent covariation among features within a category, and this internal structure influenced category learning. These experiments used an inferential missing features task, which has previously been shown to induce learning of internal category structure (Yamauchi \& Markman, 1998; Anderson et al., 2002; Chin-Parker \& Ross, 2002; 2004; Markman \& Ross, 2003). However, it is unclear whether humans can learn the internal correlational structure of a category when the structure is incidental to the task (Erikson et al., 2005). Humans have been shown to be sensitive to statistical structure even when the statistical structure is embedded in a stream of stimuli and irrelevant to the explicit task. We predict that this domain-general statistical learning translates into the realm of category learning, and Experiment 3 was designed to test whether humans can learn the internal structure of novel categories when the feature statistics are embedded in the environment and incidental to the task.

Our Modular structure contains clusters of covarying features in its periphery in addition to its set of core features, such that both core and peripheral structure can be tested. In contrast, only core structure could be tested in the Random category used in Experiments 1 and 2 because there was no peripheral structure to learn. In Experiment 3, we thus designed a Lattice structure that does contain a structured periphery, albeit with no clustering (Fig. 1C). This enabled us to evaluate the influence of coherent feature covariation on category learning while controlling for additional graph characteristics. 
In Experiment 3, we exposed participants to either a Modular or Lattice category in a temporal statistical learning (SL) paradigm with an orthogonal 1-back repeat detection task. We then tested participants' category structure knowledge in a two-alternative forced choice (2AFC) task and in an explicit feature selection task, in which they were asked to select the three features most important for the category. We again ran simulations of the neural network, exemplar, and prototype models to test whether any of these models could replicate patterns of human learning. This experiment was designed to test three predictions: (1) humans can learn internal category structure even when this structure is incidental, (2) the findings from Experiments 1 and 2 will be replicated such that it will be easier to learn the core structure of the Modular category relative to the Lattice category, and (3) the neural network model will be uniquely able to mirror patterns of human performance.

\section{Exp. 3 Behavioral Methods}

Participants. One hundred (100) participants contributed data to Experiment 3 and were recruited from Amazon Mechanical Turk (Mean age: $M=39.2, S D=10.1 ; 45 \%$ female). An additional 16 participants were excluded from analysis based on poor performance on the orthogonal task. Participants received a base pay of $\$ 2$ in addition to a bonus of up to $\$ 6$ based on performance on the orthogonal task ( $M=\$ 4.79$ bonus for 100 participants). Consent was obtained for all participants in accordance with the University of Pennsylvania IRB.

Categories and features. The beetle and butterfly species in Experiment 2 were used in Experiment 3. No catch features or category labels were used.

Category structures. Experiment 3 examined the same Modular structure from Experiments 1 and 2, but replaced the Random structure with a Lattice structure (Fig. 1C). The Lattice graph contains the same core structure as the other two graphs, but the peripheral structure differs. Like the Random graph, the Lattice graph has no clustering in its periphery. However, unlike the Random graph, the peripheral nodes are not fully connected, meaning that each peripheral node is only connected to a subset of all peripheral nodes. There were two motivations for using a Lattice instead of a Random graph: (1) the structure of the Lattice graph enables peripheral structure knowledge to be trained and tested, and (2) local node connectivity is more balanced with the Modular graph. That is, in our generated streams of 
categorical stimuli described below, each peripheral node can only be followed by three other peripheral nodes in both the Modular and Lattice structures. Comparing Modular and Lattice structures thus enables a more direct assessment of the specific influence of feature clustering on core structure learning. Assignment of species to structure and subsequent assignment of features to graph nodes was fully randomized for each participant. That is, any of the 11 species-specific features could become a core feature or a peripheral feature within a category.

Generating stimuli for the SL task. We designed an SL task that exposed participants to a category's feature co-occurrence structure by embedding co-occurrence statistics in a temporal stream of stimuli (Fig. 6A). Each SL task trial consisted of an exemplar with two features ( $\mathrm{x}$ and $\mathrm{y}$ ) that were directly connected on the graph. The subsequent trial would contain one of the same features (y) and a new feature ( $\mathrm{z}$ ), directly connected to $\mathrm{y}$. The order of the features was thus determined by a random walk across the assigned graph, with two features on the walk displayed per trial. These random walks contained 550 steps (i.e., stimuli) and were generated based on transitional probability matrices that maintained the underlying feature-based Modular and Lattice structures. In both Modular and Lattice categories, each peripheral feature could be followed by three other peripheral features (in addition to the three core features). In order to ensure that other various properties of the random walks were matched across Modular and Lattice structures, we first generated a set of 100 walks per graph which all contained balanced frequencies of core and peripheral features. All paths contained a total of 273277 presentations of core features (Lattice: $M=274.8$; Modular: $M=274.9 ; t(122)=0.36, p=0.72)$. In the set of Modular walks, we balanced the frequency of module1 and module2 features such that features from each module were seen 135-140 times. From this set of possible paths, we subsampled 62 paths for each graph structure such that Lattice and Modular structures were exactly matched on the number of 1-back repeat trials $(M=79.2, S D=6.7)$. To ensure that the feature transition statistics in these walks mirrored the intended Modular and Lattice structures, we used the feature transition statistics within each of the final walks to derive the actual joint feature probabilities for each structure (Fig. 6BC). We calculated the modularity $(Q)$ of these derived graphs to ensure that the structures underlying the Modular walks were indeed more modular than the structures underlying the Lattice walks (Fig. 6D). Modularity was calculated using the Brain Connectivity Toolbox (brain-connectivity-toolbox.net; Rubinov \& Sporns, 2010). 
Generating stimuli for the $2 A F C$ task. For each category structure, we generated 24 correct, structureconsistent exemplars with 6 features each. Each of these correct exemplars was paired with an incorrect, structure-inconsistent exemplar, in which one of the correct exemplar's features was swapped for another feature that violated the assigned category structure (Fig. 6E). 


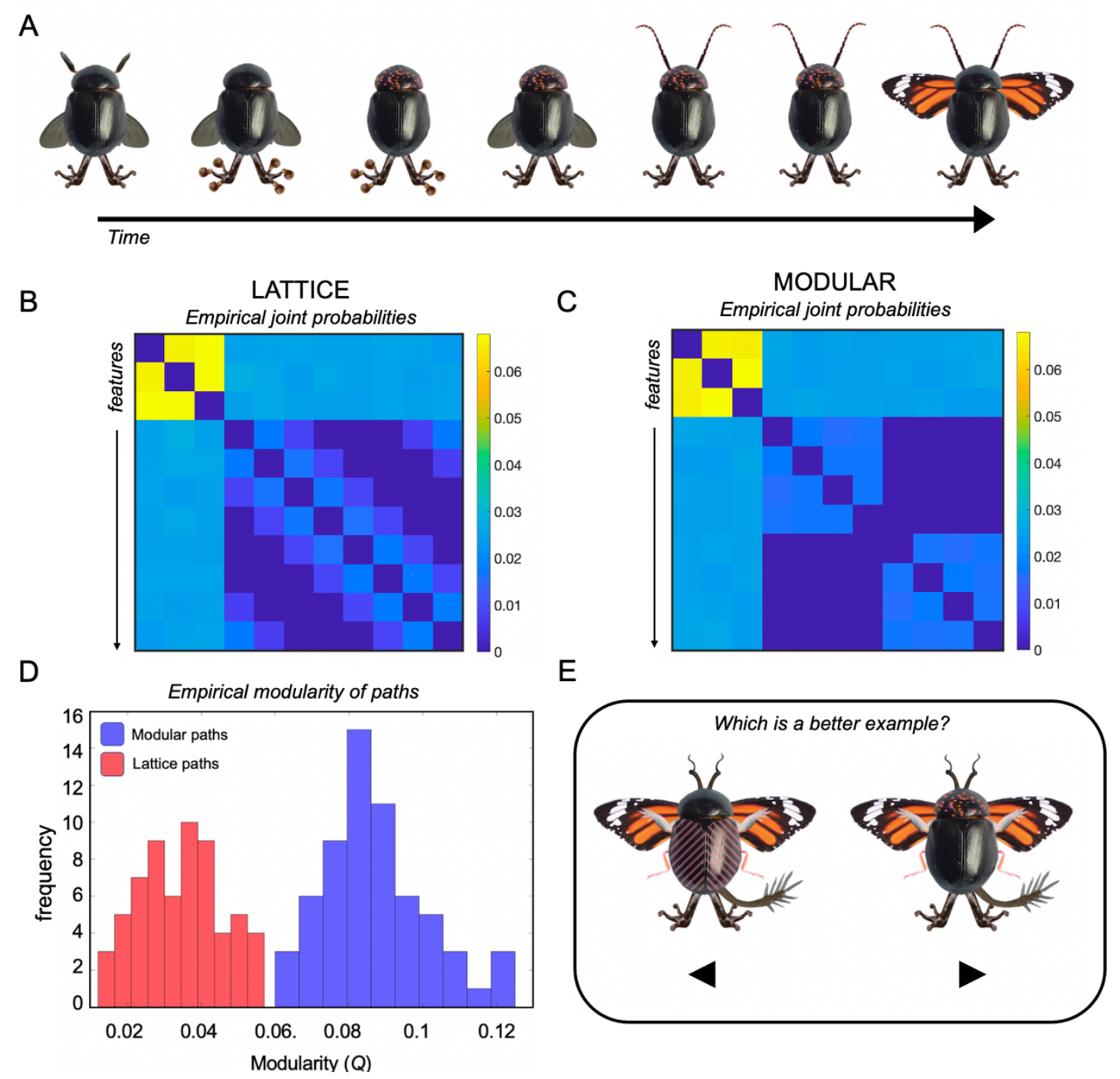

Figure 6: Experiment 3 tasks and design. Participants were exposed to a single category with either a Lattice or Modular structure. (A) In the SL task, participants viewed a stream of 550 2-feature exemplars. On each trial one feature was swapped for another and the order of features was determined by a random walk over the category structure graph. Category structure was incidental to the orthogonal 1-back repeat detection task. The actual joint probabilities between features successfully reflected the (B) Lattice and (C) Modular structures. (D) The modularity distinction between structure conditions was successfully maintained in the stimulus streams. (E) After the SL task participants completed a 2AFC task, in which they were asked to select which of two exemplars was a better example of the learned category.

Using 6-feature exemplars, the Lattice category structure allows for 24 unique correct exemplars and the Modular category structure allows for 8 unique correct exemplars; to match the number of exemplars across categories, three sets of the 8 unique Modular exemplars were used. Within each category, each of the 24 correct exemplars was assigned to test either core (8) or peripheral (16) structure knowledge. For each correct exemplar, the exact feature that was replaced to create the 
paired incorrect exemplar depended on the kind of structure (i.e., core, peripheral) the pair was designed to test. We will refer to these different correct/incorrect exemplar pairs as "Core pairs" and "Peripheral pairs".

In Core pairs, the incorrect exemplar was created by replacing one of the three core features in the correct exemplar with a peripheral feature that did not violate the peripheral structure. For example, in a Modular category exemplar containing features from module1, a core feature would be replaced with another module1 feature. Thus, the features of the incorrect exemplar are consistent with the category's peripheral structure, while the core structure is violated. A similar strategy was used to generate Core pairs for the Lattice category.

In Peripheral pairs, the incorrect exemplar was created by replacing one of the three peripheral features in the correct exemplar with another peripheral feature that did violate the peripheral structure. For example, a module1 feature might be replaced with a module2 feature; since module1 and module2 features never co-occur, the peripheral structure is violated while the core structure remains intact. A similar strategy was used to generate Peripheral pairs for the Lattice category, in which all incorrect exemplars contained a feature pair with $0 \%$ joint probability, never appearing together in the SL task.

The exemplar pairs were designed such that feature frequencies were balanced within Modular and Lattice categories. Across all exemplar pairs within each category structure, each peripheral feature appeared 9 times in correct exemplars and 10 times in incorrect exemplars; each core feature appeared 24 times in correct exemplars and 21-22 times in incorrect exemplars. The specific consistent features that were swapped with inconsistent features were also balanced: in both category structures, each peripheral feature was the consistent feature twice (i.e., the feature removed in the incorrect exemplar) and appeared three times as the inconsistent feature in incorrect exemplars. Each core feature was the consistent feature 2-3 times; core features were never used as inconsistent features.

Task protocols. Each participant was exposed to a single category whose species (i.e., Beetle or Butterfly) and structure (i.e., Modular or Lattice) was randomly assigned. The assignment of specific features to graph nodes was also randomized for each participant. Participants were told that they were part of a scientific research team that had discovered a new insect species, and that "the overall goal of the project is to better understand the characteristics and features that define this new species." However, 
participants were also informed that "the collected specimens are old and sometimes they are missing body parts (e.g., wings, legs) or their color markings have faded." As an initial exposure to the category, the participants were shown four full category exemplars, each with six features that were consistent with the assigned category structure. The participants then completed three tasks: (1) an SL task, (2) a 2AFC task, and (3) an explicit feature selection task.

Statistical learning (SL) task. Participants viewed a stream of "partial" category exemplars with two features each (Fig. 6A). The insect base remained identical throughout the experiment, but one of the two features was replaced by another feature on each subsequent trial based on one of the random walks described above; the specific walk shown to each participant was chosen randomly out of the 62 options. The SL task comprised 550 trials: in each trial the exemplar remained on screen for 1800 $\mathrm{ms}$, followed by a $200 \mathrm{~ms}$ inter-stimulus interval (ISI) with a blank screen. The participants performed an orthogonal 1-back task in which they pressed a key on each trial to indicate whether the exemplar on that trial was identical to the exemplar on the previous trial; participants were instructed to press the right arrow key for repeats and the left arrow key otherwise. Repeat exemplars were presented on $\sim 14 \%$ of the trials. Feedback was given for hits, misses, and false alarms: a green star appeared beneath the exemplar on a correct hit and a red cross appeared for misses and false alarms. A d-prime sensitivity measure was calculated and updated on each trial, transformed into a percentage score (0\%-100\%), and displayed beneath the insect stimuli for the entirely of the SL task to motivate participants to pay attention to the stimuli and successfully complete the 1-back task. The participants were told ahead of time that their final score on the last SL trial determined their bonus payment. A set of practice trials using abstract shapes was given to participants before the main SL task to acclimate them to the task and clarify instructions.

Two-alternative forced choice ( $2 A F C)$ task. In the $2 \mathrm{AFC}$ task, participants were instructed to use their new knowledge of the species to decide what full specimens should look like. Specifically, they were asked to decide on each trial which of the two specimens is a better example of the newly discovered insect (Fig. 6E). Each of the 24 trials consisted of one of the Core or Peripheral exemplar pairs described above, in which one exemplar was consistent with the category's feature-based structure and the other was inconsistent. The two exemplars were shown side by side in the center of a white screen; left/right placement of correct/incorrect exemplars was randomized on each trial. Stimuli remained on the screen until participants made a response by pressing the left or right button on their keyboard. The 
category stimuli disappeared from the screen $500 \mathrm{~ms}$ after a response, and $1000 \mathrm{~ms}$ later the next trial began.

Explicit structure task. In the final task, participants were asked to "click on the 3 features that are most important for this species." The 11 category features were presented individually, each one attached to the greyscale insect base. The 11 features appeared in a grid on the screen, and participants were asked to click on three features to make their response. When a feature was selected, a blue outline appeared around the feature so the participants could keep track of their responses. Once three features were selected, the task immediately ended.

Statistical analysis. In the SL task data, we analyzed participants' repeat detection sensitivity in order to exclude participants who did not pay sufficient attention to the stimuli. We used a d-prime sensitivity measure, in which sensitivity was defined in terms of the hit rate and false alarm rate. We excluded participants whose sensitivity score was below $50 \%$ by the final trial, resulting in 16 participants (separate from the final $N=100$ ) that were excluded from subsequent analyses.

We analyzed reaction time (RT) and accuracy on the 2AFC task. We used RT to exclude trials in which participants' responses were unlikely to be meaningful (RT $<250$ ms or RT $>10000$ ms). In the remaining trials, we determined whether participants chose the correct (1) over the incorrect exemplar (0). We averaged these accuracy scores separately for Core and Peripheral structure trials, resulting in a mean Core accuracy and mean Peripheral accuracy for each participant. Independent t-tests were used to compare mean Modular vs. Lattice core structure accuracy, and Pearson correlations were used to assess relationships between conditions.

In the Explicit structure task, we calculated accuracy based on the number of correct core features each participant selected (0-3) and compared it against chance-level performance (0.27). Independent t-tests were used to compare accuracy between Modular and Lattice structures. 
A

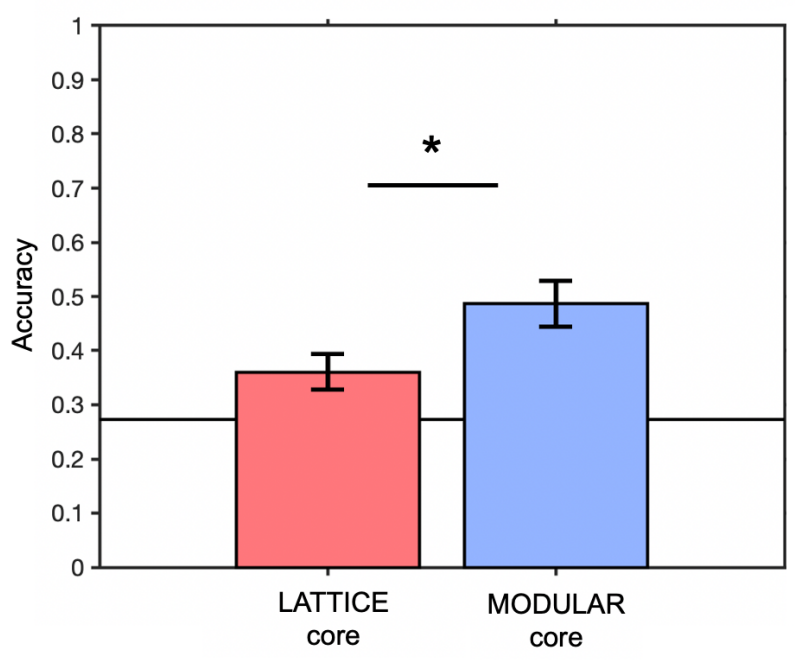

B

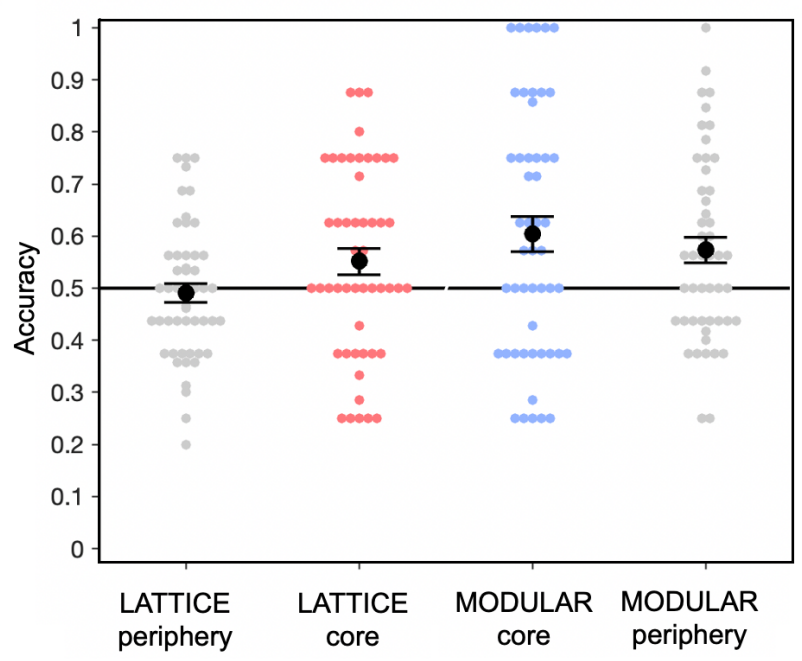

Figure 7: Behavioral results from Experiment 3. (A) In the explicit feature selection task, participants found it easier to select the three core features from the Modular vs. the Lattice category $t(98)=2.3, p=0.02$ ), replicating the preference observed in Experiments 1 and 2. (B) The 2AFC task did not reveal a significant difference in accuracy between Modular core and Lattice core trials.

\section{Exp. 3 Behavioral Results}

Accuracy on $2 A F C$ task. We determined whether participants successfully learned core and peripheral structures of Lattice and Modular categories using data from the 2AFC task (Fig. 7B). While participants' ability to learn Lattice core structure was inconclusive $(M=55 \% ; S D=18.1 \% ; t(49)=2.0$, $p=0.05)$, accuracy for Modular core structure was reliably above chance $(M=60.4 \% ; S D=24.1 \%$; $t(49)=3.1, p=0.004)$; however, there was no reliable difference between Lattice and Modular core accuracy $(t(98)=1.2, p>0.2)$. Our data also suggest that participants were not able to learn the Lattice periphery structure $(M=49.1 \% ; S D=12.9 \% ; t(49)=0.5, p>0.6)$, but were successful at learning Modular periphery structure $(M=57.4 \% ; S D=17.6 \% ; t(49)=2.9, p=0.005)$; accuracy was significantly higher for Modular vs. Lattice periphery structure $(t(49)=2.7, p=0.01)$. No reliable relationship was found between core and periphery accuracy in either the Lattice $(r(49)=0.11, p>0.4)$ or Modular $(r(49)=0.14$, $p>0.3)$ category. 
Accuracy on explicit feature selection task. We also examined core structure learning in the explicit feature selection task, in which participants selected the three features that they thought were most important for the category (Fig. 7A). Accuracy was reliably above chance for both Lattice $(t(49)=2.7, p=0.01)$ and Modular $(t(49)=5.0, p<0.00001)$ categories. Consistent with results from the previous behavioral experiments, accuracy was significantly higher for Modular relative to Lattice core structure $(t(98)=2.3$, $p=0.02)$.

\section{Exp. 3 Modeling Methods}

\section{Neural Network Model}

Model architecture and parameters. The model used in Experiment 1 was modified slightly for Experiment 3. The input and output layers were restricted to 11 features each since the model was trained to learn only one category at a time. We also removed the inhibition on the output layer so that the model was able to activate all features simultaneously despite learning to only activate two features during training (see below).

Training protocol. The model was trained on the 62 Lattice and 62 Modular paths generated for the SL task, resulting in 62 Lattice and 62 Modular simulations. Training consisted of 550 trials in which the model was presented with two features on the input layer and learned to replicate the same two features on the output layer. The training trials were presented in the same order shown to human participants.

Testing protocol. Testing trials were analogous to the $2 \mathrm{AFC}$ trials shown to human participants. On each of the 24 trials, the model was presented with the five features shared by the correct and incorrect exemplars. We observed the resulting activity on the output layer to determine whether the correct exemplar's extra feature (structure-consistent) or the incorrect exemplar's extra feature (structureinconsistent) was more strongly activated. Binary accuracy was assigned on each trial based on whether the consistent (1) or inconsistent (0) feature was more strongly activated.

Model assessment. We used the 62 Modular and 62 Lattice paths to train and test the models, resulting in 62 simulations per structure. For each structure, and within each simulation, we calculated the mean 
accuracy separately for core structure and peripheral structure trials. Accuracy was compared against chance (0.5) and independent t-tests were used to assess differences in core and peripheral structure learning across structure conditions.

Analyzing feature representations. After the full training protocol, the model was probed with each of the 11 category features by setting each individual feature unit's activation to 1 on the input layer. Settled activation in the $20 \times 20$ hidden layer was extracted, resulting in 11 final feature representations. We assessed feature similarity using Euclidean distance and used multi-dimensional scaling to visualize the space of learned feature representations.

\section{Exemplar Model}

The exemplar model used in Experiment 3 is nearly identical to the one described above. The exemplars and test stimuli are 11-feature binary vectors, except now the stored category exemplars correspond to the set of 2-feature stimuli presented during the SL task, and the test stimuli correspond to the exemplars presented in the 2AFC task. On each trial, category match $P$ for the correct and incorrect $2 \mathrm{AFC}$ test stimuli was calculated based on the summed similarity between each test stimulus and the 550 category exemplars. On each trial, match values for the correct $\left(P_{0}\right)$ and incorrect $\left(P_{1}\right)$ stimuli were compared; if $P_{0}>P_{1}, a c c=1$. In order to analyze model performance at a more finegrained level, we also calculated a non-binary measure of accuracy reflecting the difference in match values between the correct and incorrect test stimuli $\left(P_{0}-P_{1}\right)$. Mean accuracy was calculated within each of 62 simulations within each category structure, corresponding to the 62 Lattice and 62 Modular paths used in the behavioral experiment.

\section{Prototype Model}

The prototype model was almost identical to the exemplar model, except the category match $P$ for each $2 \mathrm{AFC}$ test stimulus was determined based on the Euclidean similarity of the stimulus to the centroid of all of the SL exemplars. 
A

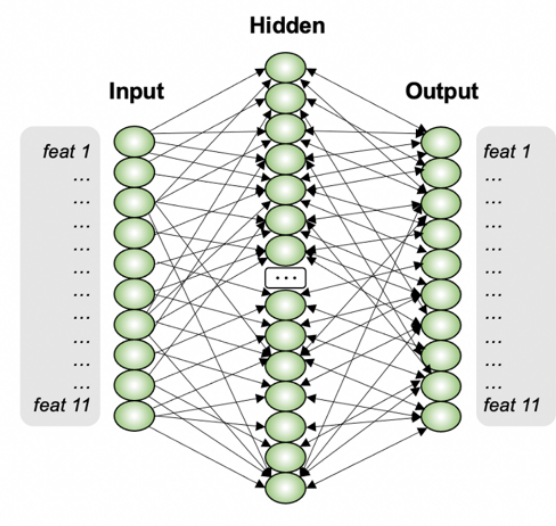

C

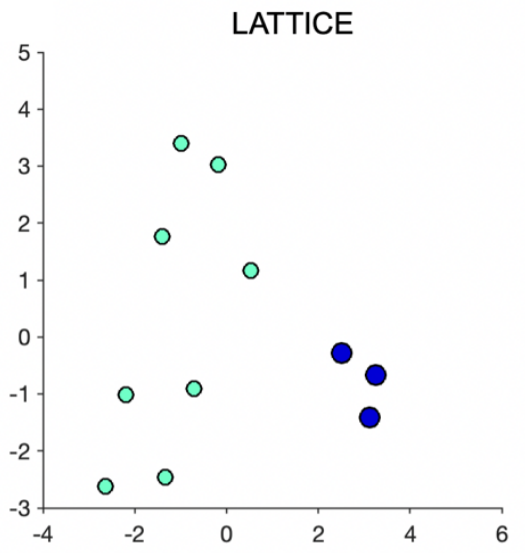

B

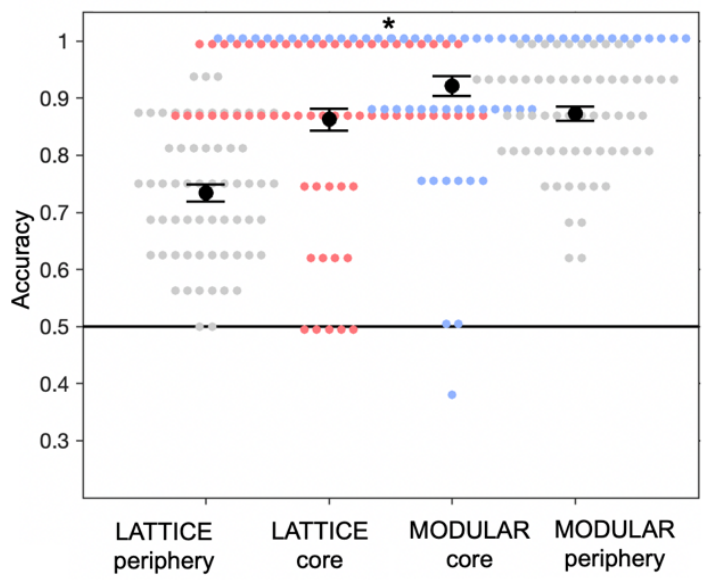

MODULAR

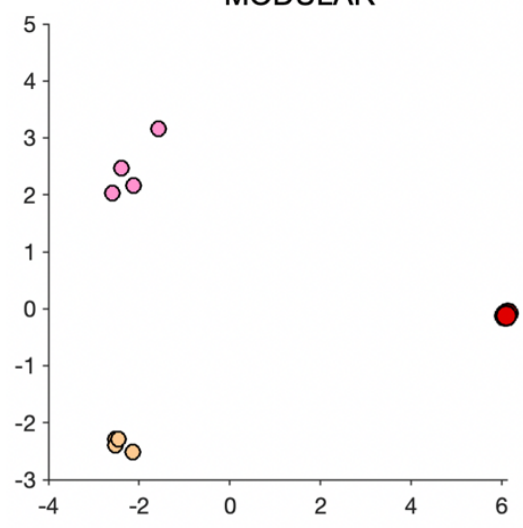

Figure 8: Neural network model simulations in Experiment 3. (A) ' l he model architecture contained 11 teature units on the input and output layers and a 400-unit hidden layer. (B) After training on the SL stimuli, the model performed more accurately on Modular core vs. Lattice core $2 \mathrm{AFC}$ trials $(t(122)=2.3, p=0.024)$. (C) Multidimensional scaling solutions for the final feature representations in the Lattice and Modular categories. Lattice core features are blue; Modular core features are red.

\section{Exp. 3 Modeling Results}

Neural network model. Mean model accuracy across runs for Lattice and Modular categories is shown in Fig. 8B. While human participants were able to learn the Modular but not the Lattice periphery structure, the model was able to learn the peripheral structure of both the Modular $(\mathrm{M}=87.3 \%$; $\mathrm{SD}=9.6 \% ; t(61)=30.6, p<0.0001)$ and Lattice categories $(\mathrm{M}=73.3 \% ; \mathrm{SD}=11.7 \% ; t(61)=15.7$, $p<0.0001)$. The model also learned the core structure of the Modular $(\mathrm{M}=92.1 \%$; $\mathrm{SD}=13.4 \%$; 
$t(61)=24.7, p<0.0001)$ and Lattice $(\mathrm{M}=86.3 \% ; \mathrm{SD}=15.0 \% ; t(61)=19.1, p<0.0001)$ categories at above chance levels. Most notably, model accuracy for Modular core structure was greater than accuracy for Lattice core structure $(t(122)=2.3, p=0.024)$. We thus replicated the Modular core benefit observed in the explicit feature selection task with human participants.

Multi-dimensional scaling solutions to the final learned feature representations are visualized in Fig. 8C. On visual inspection, core features are separated from peripheral features in both categories; we quantified this measure by calculating the ratio of mean core-peripheral feature distances to mean core-core distances within each structure, revealing the relative distance between core and peripheral features within Euclidean space. This relative distance measure was significantly higher for Modular relative to Lattice categories $(t(122)=18.0, p=<0.0001)$, demonstrating that the learned representations were again sensitive to the coherent covariation in the modules of the Modular category.

Exemplar model. Like the neural network model, the exemplar model $(c=1.8)$ was able to learn the core and peripheral structure of Lattice and Modular categories when accuracy was analyzed as a binary measure (Fig. 9A). The model performed significantly above chance on both the Lattice ( $M=70 \%$; $S D=7.0 \% ; \mathrm{p}<0.0001)$ and Modular $(M=82.3 \% ; S D=7.0 \% ; p<0.0001)$ peripheral trials, and achieved ceiling performance for Lattice and Modular core trials $(M=100 \%)$. In order to determine whether any differences could be found between Lattice and Modular core structure learning, we analyzed the non-binary accuracy data for a more sensitive measure of model performance (Fig. 9B). Here we did find a significant difference between Lattice $(M=14.5 ; S D=0.24)$ and Modular $(M=14.3 ; S D=0.23)$ core structure learning, but in the opposite direction to the human and neural network model results: the exemplar model learned the core structure significantly better in Lattice relative to Modular categories $(t(122)=4.06, p<0.0001)$. This difference increased with increasing values of the sensitivity parameter (Supplementary Fig. 1b).

Prototype model. The prototype model also was at ceiling at learning Lattice (100\%) and Modular (100\%) core structure when using the binary accuracy measure, with no difference between the conditions (Fig. 9C). However, unlike the exemplar model, the prototype model was not able to learn the peripheral structure of either the Lattice $(M=48.5 \% ; S D=6.0 \%)$ or Modular $(M=48.1 \% ; S D=7.0 \%)$ categories. The same pattern of results emerged using the more sensitive non-binary accuracy measure. 

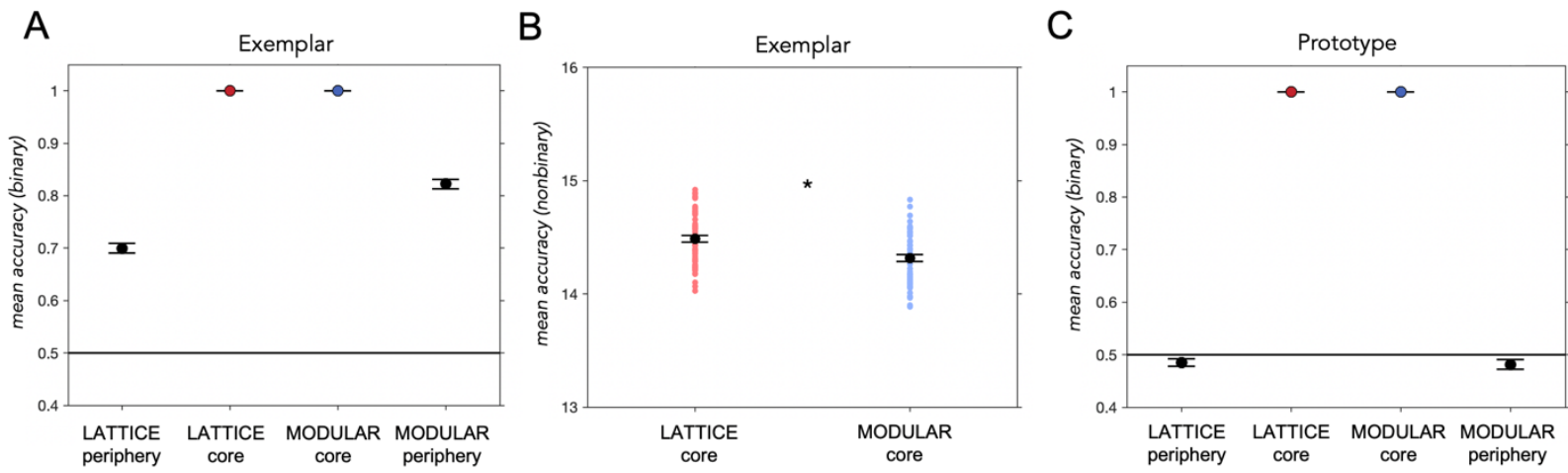

Figure 9: Exemplar and prototype simulations in Experiment 3. (A) The exemplar model successfully learned the core and peripheral structure of both the Lattice and Modular categories, but no difference in accuracy was observed between the core conditions using a binary accuracy measure. (B) Using a more sensitive non-binary accuracy measure, the exemplar model revealed increased performance on Lattice core vs. Modular core trials, contrasting with the pattern of human performance. (C) The prototype model was able to learn the core structure of both Lattice and Modular categories, but was unable to learn peripheral structure in either category.

\section{Exp. 3 Discussion}

In Experiment 3, we replicated the Modular core benefit observed in Experiments 1 and 2 in a different task paradigm and with a new graph structure. Participants were each exposed to either a Modular or Lattice category in a temporal statistical learning paradigm in which category structure was incidental to the orthogonal 1-back repeat detection task. Participants subsequently completed a 2 AFC task which tested their ability to detect structure-consistent exemplars, and an explicit feature selection task which tested whether participants had learned the three core category features.

The 2AFC results demonstrated that participants successfully learned Modular core structure, while ability to learn Lattice core structure was inconclusive. Modular core accuracy was not significantly greater than Lattice core accuracy in this case, as it was in the prior experiments. However, the results from the explicit feature selection task replicate the Modular core benefit observed in Experiments 1 and 2. Participants were able to correctly select the three core features from both the Lattice and Modular categories at above chance levels. However, accuracy was significantly higher for participants 
who were exposed to a Modular category. That is, the clustering of peripheral features in the Modular category, relative to Lattice, made it easier to learn the category's core features.

The results of Experiment 3 thus replicate the Modular core benefit observed in Experiments 1 and 2, while removing the alternative explanations discussed above. Replacing the Random graph with the Lattice graph made the peripheral structures between the two conditions more comparable, and it additionally enabled us to test core and peripheral structure in both categories with feature frequencies balanced throughout. Increased clustering in novel categories thus appears to benefit category learning across stimulus modalities and experimental tasks.

We also found that a neural network model was able to successfully replicate the human behavioral effects. The model was exposed to the same pairs of features presented to human participants in the SL task, and a subsequent test analogous to the human 2AFC task revealed that the model was more likely to generate correct relative to incorrect exemplars. The exemplar and prototype models were unable to replicate this Modular core benefit. In fact, the only difference between Modular and Lattice core performance in the exemplar model went in the opposite direction. This result appears to be a function of the exponential decay function and sensitivity parameter, which in effect warps the similarity space on each test trial such that exemplars close to the test item have the greatest influence on model predictions. In Core Pairs, the incorrect $2 \mathrm{AFC}$ stimulus has more peripheral features than does the correct $2 \mathrm{AFC}$ stimulus, and is therefore drawn closer to the peripheral-only exemplars in similarity space, which adds a source of error into the model. This is true for both Lattice and Modular categories, but this bias is even stronger for the Modular category due to the high clustering of peripheral-only exemplars within a specific module in similarity space. This is a small effect that does not affect binary accuracy and disappears at lower sensitivity parameter values (Supp. Fig. 1B). Nevertheless, it demonstrates the inability of the exemplar model to predict the Modular core benefit. These findings suggest that the Modular core benefit observed in Experiments 1, 2, and 3 emerges from a shared mechanism sensitive to clustered co-occurrence statistics across episodes, regardless of stimulus modality or task design. 


\section{General Discussion}

Across three experiments, we aimed to determine whether humans are sensitive to the coherent feature structure within individual categories and whether this aids learning. To do this, we designed novel categories characterized by different graph structures, such that some categories contained multiple communities of reliably co-occurring features (Modular) whereas other categories did not (Random, Lattice). Existing work on semantic learning demonstrates that neural network models can build distributed semantic representations that capture real-world semantic similarities and hierarchies, and that these models' success can be explained by their sensitivity to the coherent covariation of features across items (Rogers \& McClelland, 2004; McClelland \& Rogers, 2003). Here we explored whether humans can quickly build sensitivity to coherent covariation across features within a novel category. We predicted that categories with clusters of reliably co-occurring features should be easier to learn both for humans and for a neural network model simulating the tasks. We also predicted that standard models of category learning (i.e., exemplar, prototype), which do not build rich representations of correlational structure, would not be able to account for the patterns of human learning. We found support for these predictions in an inferential missing feature task, a statistical learning task, and corresponding model simulations. People are indeed sensitive to the correlational structure of individual novel categories and leverage this structure to aid learning; the unique success of the neural network model suggests that this effect depends on the rapid formation of rich distributed representations.

\section{Structure learning across domains}

Our observed benefit for Modular categories adds to our understanding of how humans extract structure from their environment. Outside of the semantic domain, recent work has highlighted humans' sensitivity to structure, characterized by underlying graph structures like the ones used here (Schapiro et al., 2013; Karuza et al., 2019; Kakaei et al., 2021; Lynn et al., 2020; Lynn \& Bassett, 2020). Karuza et al., (2019) use novel visual stimuli in a series of behavioral experiments to reveal that humans are sensitive to graph structures containing a range of different numbers and sizes of communities, or modules. A recent behavioral study (Kakaei et al., 2021) suggests that temporal community structure accelerates recognition learning of novel visual objects and affects the order in which objects are learned. Using a sequential motor task in humans, Lynn et al. (2020) utilize random walks across different graph structures to reveal overall faster responses on a modular graph relative to a lattice 
graph. Additional behavioral work suggests that humans can extract higher-order structure from the environment and leverage this learned structure to aid subsequent learning (Mark et al., 2020). The formation of potential "cognitive maps" similarly involves the extraction and representation of environmental structure that can be applied across many domains (see below). Thus, humans appear sensitive to higher-order structure, and this has implications for real-world learning in domains such as navigation, language acquisition, event learning, and knowledge accumulation (Schapiro et al., 2013; Lynn \& Bassett, 2020; Karuza et al., 2016). There also appears to be a common thread through behavioral work in humans revealing a learning benefit for modular graphs, with clusters of reliably associated nodes. Our present findings extend this phenomenon to a semantic context: humans find it easier to learn categories characterized by a modular structure containing sets of reliably co-occurring semantic features.

\section{Coherent covariation and semantic structure}

Like humans, our neural network models revealed a learning benefit for Modular categories. These findings complement previous computational investigations of semantic learning. The semantic models of Rogers \& McClelland (2004) were able to leverage coherent covariation among features to progressively differentiate semantic categories during learning in a trajectory that corresponds with the development of semantic memory in human infants. For example, animals share many features with each other (e.g., can move, has skin) that are not shared with plants. The model is presented with features of different animals and plants (e.g., bird, tree) and learns internal representations of these items across simulated developmental timescales. The model's learning algorithm drives items with shared features to share connection weights, which leads to increased similarity among animal representations (e.g., BIRD, FISH) and increased similarity among plant representations (e.g., TREE, FLOWER). The lack of shared features between animals and plants results in differentiation between these semantic classes. In other words, the coherent covariation among features enables the formation of semantic representations in the Rogers \& McClelland model that reflect the real-world semantic hierarchy. Saxe et al. (2019) formalized a notion of category coherence that describes more precisely how deep linear neural networks extract categories from noise as a function of feature co-occurrence probabilities within subsets of items. In our current studies we show that coherence can quickly govern representation building in a novel domain, in both humans and neural network models, and that this learning can reflect the structure within a category. Increased coherence within the Modular category 
input drove similarity of feature representations within modules and differentiation between modules, which led to better overall understanding of the Modular category in the models and in people.

\section{Implications for models of category learning}

Our neural network models replicated the modular benefit we observed in humans, suggesting that the learning mechanisms and representations within these models are relevant to human category learning. We believe that the model's success relies on the rich distributed internal representations that emerge during the learning process, with items overlapping in their representations as a function of feature correlation structure. This interpretation is supported by our findings that neither the exemplar nor prototype model could predict patterns of human learning. Our exemplar model was based on Nosofsky's (1986) generalized context model (GCM), which has had significant success in predicting human category learning behavior (Medin \& Schaffer, 1978; Hintzman, 1984; Nosofsky, 1984; 1998; Nosofsky et al., 2018). While the exemplar model was able to learn a category's core and peripheral structure, there was little influence of category structure on category learning. The only difference found between Modular and Lattice categories went in the opposite direction to that shown in the neural network model and human behavior, due to the way that GCM warps similarity space. The prototype model was not able to learn peripheral structure at all, and revealed no difference in core structure between categories: the averaging across features in this model completely obscured the differences in category structure. These findings reveal a qualitative deficit in these prominent category learning models. It is possible, however, that other existing category learning models could account for the findings. Here we will consider the REMERGE (Kumaran \& McClelland, 2012), ALCOVE (Krushke, 1992), and SUSTAIN (Love et al., 2004) models.

REMERGE is a neural network model built to demonstrate how inference and categorization can be accomplished by orthogonalized neural codes in the hippocampus (Kumaran \& McClelland, 2012). Its architecture contains a layer of localist feature units and a layer of localist conjunctive units; recurrent processing between these layers enables co-activation of multiple conjunctive units that code for indirectly related experiences. In the case of categorization, each training exemplar is assigned one conjunctive unit, and the features of individual test items will activate these units in proportion to their featural overlap. The retrieval dynamics in REMERGE carry out a function closely analogous to the similarity-based computation implemented in GCM (Kumaran \& McClelland, 2012, Appendix), making it likely that REMERGE would behave qualitatively similarly to the exemplar model. Indeed, 
the model shares the core characteristic of GCM: storing individual exemplars separately and avoiding an integrated representation of the kind found in neural network models with distributed representations.

ALCOVE is another neural network model implementation of an exemplar model, containing an explicit dimensional attention mechanism (Krushke, 1992). This model is similar to GCM, but errordriven weight changes between dimension units and exemplar units allows for increased attention to specific feature dimensions, and weight changes between exemplar and category units enables exemplars to differentially contribute to the category decisions. When the attention-learning rate is high, ALCOVE is sensitive to correlated feature dimensions and this can contribute to successful classification. Still, it seems likely that this model will behave qualitatively similarly to the other exemplar style models in our tasks; simulations will be needed to determine whether the attentional mechanisms may allow a better fit.

SUSTAIN is another neural network model of category learning containing a powerful dimensional attention mechanism, but does not explicitly store representations of individual items and is therefore more distinct from exemplar-style models. Rather, SUSTAIN represents categories as "clusters" within a multidimensional feature space, with new clusters formed only when existing clusters lead to large prediction errors. Love et al. (2004) specifically intended to capture category substructure, and SUSTAIN would thus likely be able to capture the structural differences between our Modular and non-Modular categories. Like ALCOVE, attentional tuning within each dimension results in sensitivity to correlated feature dimensions, which can influence category decisions. However, SUSTAIN's ability to predict our current behavioral results is unclear. Love et al. (2004) report that SUSTAIN prefers intercorrelated, versus non-intercorrelated, dimensions, but once the model learns one cluster of correlated features it finds it more difficult to learn another. This might lead to easier learning of core features in the non-Modular relative to Modular categories, the opposite of what we observed. Again, however, simulations of our current paradigms are needed to determine how SUSTAIN would fare.

All these models are neural network models in that their architectures contain layers of units which communicate with each other via weighted connections. However, the models differ substantially in their representational formats. In REMERGE and ALCOVE, exemplars are hard coded as individual 
units in the representational layer. Though SUSTAIN represents multiple categories within a single multidimensional feature space, its implementation involves individual category clusters represented in terms of their own distinct sets of feature units. In our current neural network models, features, exemplars, and categories are simultaneously represented in overlapping patterns across a single layer of hidden units. Our models do not have the bells and whistles of existing category learning models; they are simple three-layer networks intended to isolate the influence of a hidden layer employing distributed representations. We believe the empirically observed benefits for Modular categories likely requires this kind of representational format. This not only has implications for theories and models of category learning but also for the neural representations that we might expect to emerge during category learning.

\section{Potential neural substrates}

Based on prior neuroimaging investigations of statistical learning, structure learning, and category learning, we believe it is likely that our effects are underpinned by the rapid development of distributed representations in the hippocampus. There is a growing literature indicating that the hippocampus tracks information across experiences in the service of category learning (Mack et al., 2018). Evidence from neuroimaging and studies with hippocampal amnesics similarly suggests that the hippocampus is recruited for rapid statistical learning (Covington et al., 2018; Schapiro et al., 2012; 2014; 2016; TurkBrowne et al., 2009; 2010; Bornstein \& Daw, 2012; Harrison et al., 2006; Strange et al., 2005), building overlapping representations that reflect statistically strong associations between stimuli (Schapiro et al., 2012). This ability to extract commonalities across experiences is in tension with the original Complementary Learning Systems (CLS) theory, which posited that the hippocampus houses sparse, separated neural patterns which are only subsequently consolidated as distributed representations in neocortex (McClelland et al., 1995). However, we have previously proposed that the hippocampus itself contains complementary learning systems due to different representational formats in subfields CA1 vs. CA3 and Dentate Gyrus: a neural network model incorporating properties of these subfields demonstrates how sparse, separated patterns in CA3 and Dentate Gyrus that support episodic memory can coexist with distributed representations in CA1 that underlie rapid statistical learning (Schapiro et al., 2017). Our current model simulations suggest that distributed representations may be necessary to explain our category learning effects, leading us to hypothesize that these structured representations might emerge in the CA1 subfield of the hippocampus. Indeed, the models used here 
are functionally equivalent to the monosynaptic pathway of our hippocampus model — the pathway connecting entorhinal cortex with region CA1.

Hippocampal involvement in the rapid extraction of category structure would be consistent with our previous finding that the hippocampus is sensitive to graph community structure. Schapiro et al. (2016) exposed humans to a sequence of abstract visual stimuli whose order was determined by random walks across a modular graph. Each module, or community, corresponded to a group of stimuli that appeared in close temporal proximity within the experiment. After exposure, multivoxel hippocampal patterns evoked by stimuli within the same community were more similar than those from different communities, revealing sensitivity to high-level graph structure. Additional evidence of structure learning and representation in the hippocampus comes from research on "cognitive maps". Tolman (1948) introduced cognitive maps as abstract, domain-general structures that represent relations between entities. These structured representations are powerful because they can enable inference of relationships between entities that have not been directly observed, leading to generalization and improved learning performance (Behrens et al., 2018; Whittington et al., 2020). Cognitive maps are also a powerful theoretical framework because they can be used to represent relations between varied kinds of entities, such as places (O'Keefe \& Nadel, 1978; Hafting et al., 2005; Javadi et al., 2017; Killian \& Buffalo, 2018; Stachenfeld et al., 2017; Epstein et al., 2017), objects (Mark et al., 2020; Whittington et al., 2020), people (Tavares et al., 2015; Park et al., 2021), concepts (Constantinescu et al., 2016), events (Hassabis et al., 2007), and other abstract spaces (Behrens et al., 2018; Franklin \& Frank, 2018; Schuck et al., 2016; Theves et al., 2019). The medial temporal lobe (MTL) and hippocampus specifically have been implicated in representing or otherwise processing structural elements of these cognitive maps (O'Keefe \& Nadel, 1978; Hafting et al., 2005; Javadi et al., 2017; Killian \& Buffalo; Stachenfeld et al., 2017; Tavares et al., 2015; Park et al., 2021; Constantinescu et al., 2016; Hassabis et al., 2007; Theves et al., 2019). Evidence thus suggests that graph or map-like structures rely at least in part on hippocampal representations, reinforcing the hypothesis that hippocampal representations underlie our observed effects of graph structure on category learning.

While the rapid learning capabilities of the hippocampus would facilitate structure learning in novel domains like the ones employed here, we would expect a consolidated form of this knowledge to rely primarily on neocortical areas that are known to support long-term conceptual knowledge, like the 
anterior temporal lobes (Patterson et al., 2007; Lambon Ralph et al., 2010; 2017; Peelen \& Caramazza, 2012).

\section{Relational structure within real-world concepts}

We have demonstrated how statistical relations between features-above and beyond the specific features themselves_ — can benefit category learning. These results can be considered through the lens of relational knowledge, which plays a significant role in the construction of conceptual theories. While some "entity" concepts can more easily be defined in terms of particular features (e.g., apple, beetle), other "relational" concepts are more easily defined in terms of particular relations between entities in the world (e.g., bridge, friend; Gentner \& Kurtz, 2005; Asmuth \& Gentner, 2017). The meaning of a relational concept comprises a certain relation between elements, rather than specific sensorimotor features. For example, a bridge is a structure connecting two entities—shape, material, and size features can be irrelevant for categorization. Indeed, a bridge might not be a physical object at all, but rather an abstract connection between two separate ideas. Relationality has consequences for how concepts are processed. For example, it is easier to imagine an ideal example of a relational concept relative to an entity concept (Goldwater et al., 2011), and relational concepts may be more semantically mutable across contexts than entity concepts (Asmuth \& Gentner, 2017). Further, relational structure within different concepts or domains drives analogical reasoning (Gentner et al., 1993; Holyoak, 2012). Relationality is associated with conceptual abstractness, since both terms characterize concepts that are perhaps more appropriately or efficiently represented in terms of non-tangible, rather than concrete, features.

Feature relations within concepts have been considered in the context of "category coherence" in prior behavioral work (Murphy \& Medin, 1985; Wisniewski, 1995; Spalding \& Murphy, 1996; Rehder \& Ross, 2001). This body of work considers "coherence" to reflect known semantic, thematic, or causal relations between features that participants bring with them into an experimental setting. In other words, coherent categories are those whose feature combinations make sense in light of prior knowledge (e.g., has wings, can fly). Prior knowledge can affect interpretations of features during category processing and learning (Wisniewski \& Medin, 1994; Spalding \& Ross, 2000; Spalding \& Murphy, 1996) and can make it easier to integrate features during category learning (Murphy \& Allopenna, 1994). Incidentally, it has been reported that similarity-based category learning modelslike the exemplar and prototype models implemented here-cannot account for the acquisition of 
abstract, coherent categories (Erikson et al., 2005). In sum, it is easier to learn a new category when it contains sets of features that are known to be associated, or correlated, with each other in the real world. Correlated features in turn play an important role in relational category learning (Goldwater et al., 2018).

The novel insects learned in our current experiments did not contain rich semantic, thematic, and causal relations - indeed, our feature combinations could be considered incoherent with respect to prior knowledge (e.g., antennae, claws). The structure embedded within each of our insect categories relied on feature correlations that were not imported from the real world but were learned online in the context of the experiment. Nevertheless, we replicated the finding that feature correlations benefit learning. While this is consistent with the category learning research summarized above, we need not invoke prior knowledge to explain our observed effect. Feature correlations and category coherence benefit learning even in the absence of prior knowledge. Our findings thus suggest how, without any prior semantic knowledge, observed feature correlations can aid concept learning de novo and provide the scaffolding for relational meaning. More generally, representing concepts in terms of specific features as well as feature relations_ — and understanding how these feature relations are learned_-may provide traction on understanding "relational" or "abstract" concepts that tend to be empirically elusive.

\section{Representation of abstract structure}

Our behavioral data reveal an effect of category structure on category learning, and our simulations suggest that distributed representations underlie this effect. However, we do not know whether the representations built during category learning are representations of structure per se. The effects of graph structure we observe here might be a result of feature-, exemplar-, or category-specific representations becoming more or less similar to each other during learning depending on their patterns of co-occurrence. Indeed, this is how the neural network models work. It is possible, though, that category learning can result in abstracted representations of structure that exist independently from the feature representations themselves.

A theory of abstract, explicit structure representations has been put forward by Kemp, Tenenbaum, and colleagues (Kemp \& Tenenbaum, 2008; Tenenbaum et al., 2011). In their view, structure does not emerge bottom-up within a distributed representational system, but is instead discovered by an 
algorithm that performs probabilistic inference over explicit graph representations and graph-building grammars. While this account of structure learning contrasts with the mechanisms that allow our neural network model to account for our current findings, there are demonstrations that neural network models can build representations that have this abstract—if not explicit—quality, for example learning representations that reflect the abstract structure of a family tree (Rogers \& McClelland, 2008; Hinton, 1986).

Abstract representations of structure can be empirically tested using a "structure transfer" paradigm. Mark et al. (2020) implemented such a paradigm to determine whether representations of structure are formed and whether they aid future learning. The researchers exposed participants to an environment defined either by a lattice or modular graph structure; visual images of real-world objects were assigned to different graph nodes, and the presence of graph edges indicated a possible temporal transition between these objects. After learning this initial environment, participants were introduced to a second environment structured according to the same modular graph but defined by a completely new set of visual objects. The researchers observed that participants who were initially exposed to a modular environment found it easier to learn a second modular environment, even though the environments had no visual objects in common. This suggests that participants not only learned the structure of the environment, but transformed it away from the environment's specific features and represented it in an abstract form that could then be applied to future learning environments (Mark et al., 2020). In the category learning context, future work could test whether learning a category characterized by a certain graph structure makes it easier to learn a second category with the same structure, even when the categories share no features in common. This would help clarify to what extent the brain learns representations of the category structure separate from the associations between particular category features.

\section{Conclusions}

We presented three human behavioral experiments and corresponding model simulations to test the influence of category structure on category learning. We found that humans find it easier to learn categories containing sets of reliably co-occurring features, and that this learning is likely underpinned by rapidly formed distributed representations. 


\section{References}

Anderson, A. L., Ross, B. H., \& Chin-Parker, S. (2002). A further investigation of category learning by inference. Memory \& Cognition, 30(1), 119-128.

Asmuth, J., \& Gentner, D. (2017). Relational categories are more mutable than entity categories. Quarterly Journal of Experimental Psychology, 70(10), 2007-2025.

Behrens, T. E., Muller, T. H., Whittington, J. C., Mark, S., Baram, A. B., Stachenfeld, K. L., \& Kurth-Nelson, Z. (2018). What is a cognitive map? Organizing knowledge for flexible behavior. Neuron, 100(2), 490-509.

Bornstein, A. M., \& Daw, N. D. (2012). Dissociating hippocampal and striatal contributions to sequential prediction learning. European Journal of Neuroscience, 35(7), 1011-1023.

Bowman, C. R., Iwashita, T., \& Zeithamova, D. (2020). Tracking prototype and exemplar representations in the brain across learning. eLife, 9, e59360.

Bowman, C., \& Zeithamova, D. (2021). Coherent category training enhances generalization and increases reliance on prototype representations.

Chin-Parker, S., \& Ross, B. H. (2002). The effect of category learning on sensitivity to within-category correlations. Memory \& Cognition, 30(3), 353-362.

Chin-Parker, S., \& Ross, B. H. (2004). Diagnosticity and prototypicality in category learning: a comparison of inference learning and classification learning. Journal of Experimental Psychology: Learning, Memory, and Cognition, 30(1), 216.

Constantinescu, A. O., O’Reilly, J. X., \& Behrens, T. E. (2016). Organizing conceptual knowledge in humans with a gridlike code. Science, 352(6292), 1464-1468.

Covington, N. V., Brown-Schmidt, S., \& Duff, M. C. (2018). The necessity of the hippocampus for statistical learning. Journal of cognitive neuroscience, 30(5), 680-697.

Cree, G. S., McRae, K., \& McNorgan, C. (1999). An attractor model of lexical conceptual processing: Simulating semantic priming. Cognitive Science, 23(3), 371-414.

Devraj, A., Zhang, Q., \& Griffiths, T. (2021). The Dynamics of Exemplar and Prototype Representations Depend on Environmental Statistics. In Proceedings of the Annual Meeting of the Cognitive Science Society (Vol. 43, No. 43).

Epstein, R. A., Patai, E. Z., Julian, J. B., \& Spiers, H. J. (2017). The cognitive map in humans: spatial navigation and beyond. Nature neuroscience, 20(11), 1504-1513.

Erickson, J. E., Chin-Parker, S., \& Ross, B. H. (2005). Inference and classification learning of abstract coherent categories. Journal of Experimental Psychology: Learning, Memory, and Cognition, 31(1), 86.

Frost, R., Armstrong, B. C., \& Christiansen, M. H. (2019). Statistical learning research: A critical review and possible new directions. Psychological Bulletin, 145(12), 1128. 
Franklin, N. T., \& Frank, M. J. (2018). Compositional clustering in task structure learning. PLoS computational biology, 14(4), e1006116.

Gentner, D., \& Kurtz, K. J. (2005). Relational categories.

Gentner, D., Rattermann, M. J., \& Forbus, K. D. (1993). The roles of similarity in transfer: Separating retrievability from inferential soundness. Cognitive psychology, 25(4), 524-575.

Goldwater, M. B., Don, H. J., Krusche, M. J., \& Livesey, E. J. (2018). Relational discovery in category learning. Journal of Experimental Psychology: General, 147(1), 1.

Goldwater, M. B., Markman, A. B., \& Stilwell, C. H. (2011). The empirical case for role-governed categories. Cognition, 118(3), 359-376.

Hafting, T., Fyhn, M., Molden, S., Moser, M. B., \& Moser, E. I. (2005). Microstructure of a spatial map in the entorhinal cortex. Nature, 436(7052), 801-806.

Harrison, L. M., Duggins, A., \& Friston, K. J. (2006). Encoding uncertainty in the hippocampus. Neural Networks, 19(5), 535-546.

Hassabis, D., Kumaran, D., Vann, S. D., \& Maguire, E. A. (2007). Patients with hippocampal amnesia cannot imagine new experiences. Proceedings of the National Academy of Sciences, 104(5), 1726-1731.

Hinton, G. E. (1986, August). Learning distributed representations of concepts. In Proceedings of the eighth annual conference of the cognitive science society (Vol. 1, p. 12).

Hintzman, D. L. (1984). MINERVA 2: A simulation model of human memory. Behavior Research Methods, Instruments, \& Computers, 16(2), 96-101.

Holyoak, K. J. (2012). Analogy and relational reasoning. In K. J. Holyoak \& R. G. Morrison (Eds.), The Oxford bandbook of thinking and reasoning (pp. 234-259). Oxford University Press.

Javadi, A. H., Emo, B., Howard, L. R., Zisch, F. E., Yu, Y., Knight, R., ... \& Spiers, H. J. (2017). Hippocampal and prefrontal processing of network topology to simulate the future. Nature communications, 8(1), 1-11.

Kahn, A. E., Karuza, E. A., Vettel, J. M., \& Bassett, D. S. (2018). Network constraints on learnability of probabilistic motor sequences. Nature human behaviour, 2(12), 936-947.

Kakaei, E., Aleshin, S., \& Braun, J. (2021). Visual object recognition is facilitated by temporal community structure. Learning \& Memory, 28(5), 148-152.

Karuza, E. A., Kahn, A. E., \& Bassett, D. S. (2019). Human sensitivity to community structure is robust to topological variation. Complexity, 2019.

Karuza, E. A., Kahn, A. E., Thompson-Schill, S. L., \& Bassett, D. S. (2017). Process reveals structure: How a network is traversed mediates expectations about its architecture. Scientific reports, 7(1), 1-9.

Karuza, E. A., Thompson-Schill, S. L., \& Bassett, D. S. (2016). Local patterns to global architectures: influences of network topology on human learning. Trends in cognitive sciences, 20(8), 629-640.

Kemp, C., \& Tenenbaum, J. B. (2008). The discovery of structural form. Proceedings of the National Academy of Sciences, 105(31), 10687-10692. 
Killian, N. J., \& Buffalo, E. A. (2018). Grid cells map the visual world. Nature neuroscience, 21(2), 161-162.

Komatsu, L. K. (1992). Recent views of conceptual structure. Psychological bulletin, 112(3), 500.

Kruschke, J. K. (1992). ALCOVE: an exemplar-based connectionist model of category learning. Psychological review, 99(1), 22.

Kumaran, D., \& McClelland, J. L. (2012). Generalization through the recurrent interaction of episodic memories: a model of the hippocampal system. Psychological review, 119(3), 573.

Love, B. C., Medin, D. L., \& Gureckis, T. M. (2004). SUSTAIN: a network model of category learning. Psychological review, 111(2), 309.

Lynn, C. W., \& Bassett, D. S. (2020). How humans learn and represent networks. Proceedings of the National Academy of Sciences, 117(47), 29407-29415.

Lynn, C. W., Kahn, A. E., Nyema, N., \& Bassett, D. S. (2020). Abstract representations of events arise from mental errors in learning and memory. Nature communications, 11(1), 1-12.

Mack, M. L., Love, B. C., \& Preston, A. R. (2018). Building concepts one episode at a time: The hippocampus and concept formation. Neuroscience letters, 680, 31-38.

Mack, M. L., Preston, A. R., \& Love, B. C. (2013). Decoding the brain's algorithm for categorization from its neural implementation. Current Biology, 23(20), 2023-2027.

Mark, S., Moran, R., Parr, T., Kennerley, S. W., \& Behrens, T. E. (2020). Transferring structural knowledge across cognitive maps in humans and models. Nature communications, 11(1), 1-12.

Markman, A. B., \& Ross, B. H. (2003). Category use and category learning. Psychological bulletin, 129(4), 592.

McClelland, J. L., McNaughton, B. L., \& O'Reilly, R. C. (1995). Why there are complementary learning systems in the hippocampus and neocortex: insights from the successes and failures of connectionist models of learning and memory. Psychological review, 102(3), 419

McClelland, J., Rogers, T. The parallel distributed processing approach to semantic cognition. Nat Rev Neurosci 4, 310-322 (2003). https://doi.org/10.1038/nrn1076

McRae, K., Cree, G. S., Westmacott, R., \& Sa, V. R. D. (1999). Further evidence for feature correlations in semantic memory. Canadian Joumal of Experimental Psychology/Revue canadienne de psychologie expérimentale, 53(4), 360.

McRae, K., De Sa, V. R., \& Seidenberg, M. S. (1997). On the nature and scope of featural representations of word meaning. Journal of Experimental Psychology: General, 126(2), 99.

Medin, D. L., \& Schaffer, M. M. (1978). Context theory of classification learning. Psychological review, 85(3), 207.

Medin, D. L., \& Shoben, E. J. (1988). Context and structure in conceptual combination. Cognitive Psychology, 20(2), 158-190.

Medin, D. L., Wattenmaker, W. D., \& Hampson, S. E. (1987). Family resemblance, conceptual cohesiveness, and category construction. Cognitive psychology, 19(2), 242-279. 
Morton, N. W., Schlichting, M. L., \& Preston, A. R. (2020). Representations of common event structure in medial temporal lobe and frontoparietal cortex support efficient inference. Proceedings of the National Academy of Sciences, 117(47), 29338-29345.

Murphy, G. L., \& Allopenna, P. D. (1994). The locus of knowledge effects in concept learning. Journal of Experimental Psychology: Learning, Memory, and Cognition, 20(4), 904.

Murphy, G. L., \& Medin, D. L. (1985). The role of theories in conceptual coherence. Psychological review, 92(3), 289.

Nosofsky, R. M. (1984). Choice, similarity, and the context theory of classification. Journal of Experimental Psychology: Learning, memory, and cognition, 10(1), 104.

Nosofsky, R. M. (1986). Attention, similarity, and the identification-categorization relationship. Journal of experimental psychology: General, 115(1), 39.

Nosofsky, R. M. (2011). The generalized context model: An exemplar model of classification. Formal approaches in categorization, 18-39.

Nosofsky, R. M., Sanders, C. A., \& McDaniel, M. A. (2018). Tests of an exemplar-memory model of classification learning in a high-dimensional natural-science category domain. Journal of Experimental Psychology: General, 147(3), 328.

O'keefe, J., \& Nadel, L. (1978). The hippocampus as a cognitive map. Oxford university press.

O'Reilly, R. C., Munakata, Y., Frank, M. J., Hazy, T. E., and Contributors (2020). Computational Cognitive Neuroscience. Wiki Book, 4th Edition (2020). URL: https://github.com/CompCogNeuro/ed4

Park, S. A., Miller, D. S., \& Boorman, E. D. (2021). Inferences on a Multidimensional Social Hierarchy Use a Grid-like Code. bioRxiv, 2020-05.

Patterson, K., Nestor, P. J., \& Rogers, T. T. (2007). Where do you know what you know? The representation of semantic knowledge in the human brain. Nature reviews neuroscience, 8(12), 976-987.

Peelen, M. V., \& Caramazza, A. (2012). Conceptual object representations in human anterior temporal cortex. Journal of Neuroscience, 32(45), 15728-15736.

Ralph, M. A. L., Jefferies, E., Patterson, K., \& Rogers, T. T. (2017). The neural and computational bases of semantic cognition. Nature Reviews Neuroscience, 18(1), 42-55.

Ralph, M. A. L., Sage, K., Jones, R. W., \& Mayberry, E. J. (2010). Coherent concepts are computed in the anterior temporal lobes. Proceedings of the National Academy of Sciences, 107(6), 2717-2722.

Rehder, B., \& Ross, B. H. (2001). Abstract coherent categories. Journal of Experimental Psychology: Learning, Memory, and Cognition, 27(5), 1261.

Rips, L. J., Smith, E. E., \& Medin, D. L. (2012). Concepts and categories: Memory, meaning, and metaphysics.

Rogers, T. T., \& McClelland, J. L. (2004). Semantic cognition: A parallel distributed processing approach. MIT press.

Rogers, T. T., \& McClelland, J. L. (2008). Précis of semantic cognition: A parallel distributed processing approach. Behavioral and Brain Sciences, 31(6), 689-714. 
Rosch, E. H. (1973). Natural categories. Cognitive psychology, 4(3), 328-350.

Rosch, E. (1975). Cognitive representations of semantic categories. Journal of experimental psychology: General, 104(3), 192.

Rosch, E., \& Mervis, C. B. (1975). Family resemblances: Studies in the internal structure of categories. Cognitive psychology, 7(4), 573-605.

Rubinov, M., \& Sporns, O. (2011). Weight-conserving characterization of complex functional brain networks. Neuroimage, 56(4), 2068-2079.

Saffran, J. R., Aslin, R. N., \& Newport, E. L. (1996). Statistical learning by 8-month-old infants. Science, 274(5294), 1926-1928.

Saxe, A. M., McClelland, J. L., \& Ganguli, S. (2019). A mathematical theory of semantic development in deep neural networks. Proceedings of the National Academy of Sciences, 116(23), 11537-11546.

Schapiro, A. C., Gregory, E., Landau, B., McCloskey, M., \& Turk-Browne, N. B. (2014). The necessity of the medial temporal lobe for statistical learning. Journal of cognitive neuroscience, 26(8), 1736-1747.

Schapiro, A. C., Kustner, L. V., \& Turk-Browne, N. B. (2012). Shaping of object representations in the human medial temporal lobe based on temporal regularities. Current biology, 22(17), 1622-1627.

Schapiro, A. C., Rogers, T. T., Cordova, N. I., Turk-Browne, N. B., \& Botvinick, M. M. (2013). Neural representations of events arise from temporal community structure. Nature neuroscience, 16(4), 486-492.

Schapiro, A. C., Turk-Browne, N. B., Botvinick, M. M., \& Norman, K. A. (2017). Complementary learning systems within the hippocampus: a neural network modelling approach to reconciling episodic memory with statistical learning. Philosophical Transactions of the Royal Society B: Biological Sciences, 372(1711), 20160049.

Schapiro, A. C., Turk-Browne, N. B., Norman, K. A., \& Botvinick, M. M. (2016). Statistical learning of temporal community structure in the hippocampus. Hippocampus, 26(1), 3-8.

Schlichting, M. L., Guarino, K. F., Schapiro, A. C., Turk-Browne, N. B., \& Preston, A. R. (2017). Hippocampal structure predicts statistical learning and associative inference abilities during development. Journal of cognitive neuroscience, 29(1), 37-51

Schuck, N. W., Cai, M. B., Wilson, R. C., \& Niv, Y. (2016). Human orbitofrontal cortex represents a cognitive map of state space. Neuron, 91(6), 1402-1412.

Sloman, S. A., Love, B. C., \& Ahn, W. K. (1998). Feature centrality and conceptual coherence. Cognitive Science, 22(2), 189-228.

Smith, J. D., \& Minda, J. P. (1998). Prototypes in the mist: The early epochs of category learning. Journal of Experimental Psychology: Learning, Memory, and Cognition, 24(6), 1411.

Smith, J. D., \& Minda, J. P. (2002). Distinguishing prototype-based and exemplar-based processes in dot-pattern category learning. Joumal of Experimental Psychology: Learning, Memory, and Cognition, 28(4), 800.

Solomon, S. H., Medaglia, J. D., \& Thompson-Schill, S. L. (2019). Implementing a concept network model. Behavior research methods, 51(4), 1717-1736. 
Solomon, K. O., Medin, D. L., \& Lynch, E. (1999). Concepts do more than categorize. Trends in cognitive sciences, 3(3), 99-105.

Spalding, T. L., \& Murphy, G. L. (1996). Effects of background knowledge on category construction. Journal of Experimental Psychology: Learning, Memory, and Cognition, 22(2), 525.

Spalding, T. L., \& Ross, B. H. (2000). Concept learning and feature interpretation. Memory \& cognition, 28(3), 439-451.

Stachenfeld, K. L., Botvinick, M. M., \& Gershman, S. J. (2017). The hippocampus as a predictive map. Nature neuroscience, 20(11), 1643-1653.

Strange, B. A., Duggins, A., Penny, W., Dolan, R. J., \& Friston, K. J. (2005). Information theory, novelty and hippocampal responses: unpredicted or unpredictable?. Neural Networks, 18(3), 225-230.

Tavares, R. M., Mendelsohn, A., Grossman, Y., Williams, C. H., Shapiro, M., Trope, Y., \& Schiller, D. (2015). A map for social navigation in the human brain. Neuron, 87(1), 231-243.

Tenenbaum, J. B., Kemp, C., Griffiths, T. L., \& Goodman, N. D. (2011). How to grow a mind: Statistics, structure, and abstraction. science, 331(6022), 1279-1285.

Theves, S., Fernandez, G., \& Doeller, C. F. (2019). The hippocampus encodes distances in multidimensional feature space. Current Biology, 29(7), 1226-1231.

Tolman, E. C. (1948). Cognitive maps in rats and men. Psychological review, 55(4), 189.

Turk-Browne, N. B., Scholl, B. J., Chun, M. M., \& Johnson, M. K. (2009). Neural evidence of statistical learning: Efficient detection of visual regularities without awareness. Journal of cognitive neuroscience, 21(10), 1934-1945.

Turk-Browne, N. B., Scholl, B. J., Johnson, M. K., \& Chun, M. M. (2010). Implicit perceptual anticipation triggered by statistical learning. Journal of Neuroscience, 30(33), 11177-11187.

Tyler, L. K., Moss, H. E., Durrant-Peatfield, M. R., \& Levy, J. P. (2000). Conceptual structure and the structure of concepts: A distributed account of category-specific deficits. Brain and language, 75(2), 195-231.

Tyler, L. K., \& Moss, H. E. (2001). Towards a distributed account of conceptual knowledge. Trends in cognitive sciences, 5(6), 244-252.

Unger, L., Savic, O., \& Sloutsky, V. M. (2020). Simple mechanisms, rich structure: Statistical co-occurrence regularities in language shape the development of semantic knowledge. In $\operatorname{Cog} S_{c i}$.

Watts, D. J., \& Strogatz, S. H. (1998). Collective dynamics of 'small-world' networks. Nature, 393(6684), 440442.

Whittington, J. C., Muller, T. H., Mark, S., Chen, G., Barry, C., Burgess, N., \& Behrens, T. E. (2020). The Tolman-Eichenbaum machine: Unifying space and relational memory through generalization in the hippocampal formation. Cell, 183(5), 1249-1263.

Wisniewski, E. J. (1995). Prior knowledge and functionally relevant features in concept learning. Journal of Experimental Psychology: Learning, Memory, and Cognition, 21(2), 449. 
Wisniewski, E. J., \& Gentner, D. (1991). On the combinatorial semantics of noun pairs: Minor and major adjustments to meaning. In Advances in psychology (Vol. 77, pp. 241-284). North-Holland.

Wisniewski, E. J., \& Medin, D. L. (1994). On the interaction of theory and data in concept learning. Cognitive Science, 18(2), 221-281.

Yamauchi, T., \& Markman, A. B. (1998). Category learning by inference and classification. Journal of Memory and language, 39(1), 124-148. 


\section{Supplementary Materials}

\section{Feature List A}

\begin{tabular}{|l|llll|}
\hline Experimental & \multicolumn{3}{l|}{ Idiosyncratic } & \\
\hline large & eats figs & eats olives & eats cherries & eats blueberries \\
two legs & eats dates & eats papaya & eats apricots & eats clementines \\
solitary & eats plums & eats tulips & eats coconuts & eats cranberries \\
blue eyes & eats kiwis & eats lilacs & eats honeydew & eats raspberries \\
bushy tail & eats limes & eats bananas & eats pineapple & eats blackberries \\
sleeps in caves & eats mango & eats peaches & eats tangerine & eats pomegranates \\
has horns & eats pears & eats rhubarb & eats daffodils & eats strawberries \\
growls & eats roses & eats daisies & eats cantaloupe & \\
brown fur & eats apples & eats violets & eats grapefruit & \\
drinks water & eats grapes & eats orchids & eats nectarines & \\
striped & eats lemons & eats poppies & eats watermelon & \\
\hline
\end{tabular}

\section{Feature List B}

\begin{tabular}{|l|llll|}
\hline Experimental & Idiosyncratic & & \\
small & eats corn & eats turnips & eats cashews & eats asparagus \\
four legs & eats beans & eats pecans & eats peanuts & eats mushrooms \\
social & eats beets & eats avocado & eats broccoli & eats hazelnuts \\
grey eyes & eats leeks & eats arugula & eats cucumber & eats chestnuts \\
hairless tail & eats bamboo & eats cabbage & eats eggplant & eats pistachios \\
sleeps in trees & eats celery & eats carrots & eats potatoes & eats cauliflower \\
has claws & eats fennel & eats lettuce & eats pumpkins & eats horseradish \\
roars & eats ginger & eats peppers & eats radishes & \\
black fur & eats onions & eats spinach & eats shallots & \\
drinks milk & eats squash & eats almonds & eats zucchini & \\
spotted & eats tomato & eats walnuts & eats artichoke & \\
\hline
\end{tabular}

\section{Catch Features}

\begin{tabular}{|l|l|}
\hline List $A$ & List $B$ \\
\hline has fangs & has a mane \\
long ears & short ears \\
white feet & silver feet \\
short whiskers & long whiskers \\
has feathers & has wings \\
long nose & short nose \\
\hline
\end{tabular}

Supplementary Table 1: Full list of verbal features used in Exp. 1. For each participant, feature lists $\mathrm{A}$ and B were randomly assigned to the Modular and Random structures. The first three features in each list were assigned to the core nodes, and the other eight features were randomly assigned to the peripheral nodes. Idiosyncratic features were included to add variability across exemplars and were only seen once or twice by each participant. Each catch feature only appeared once in a catch trial. 


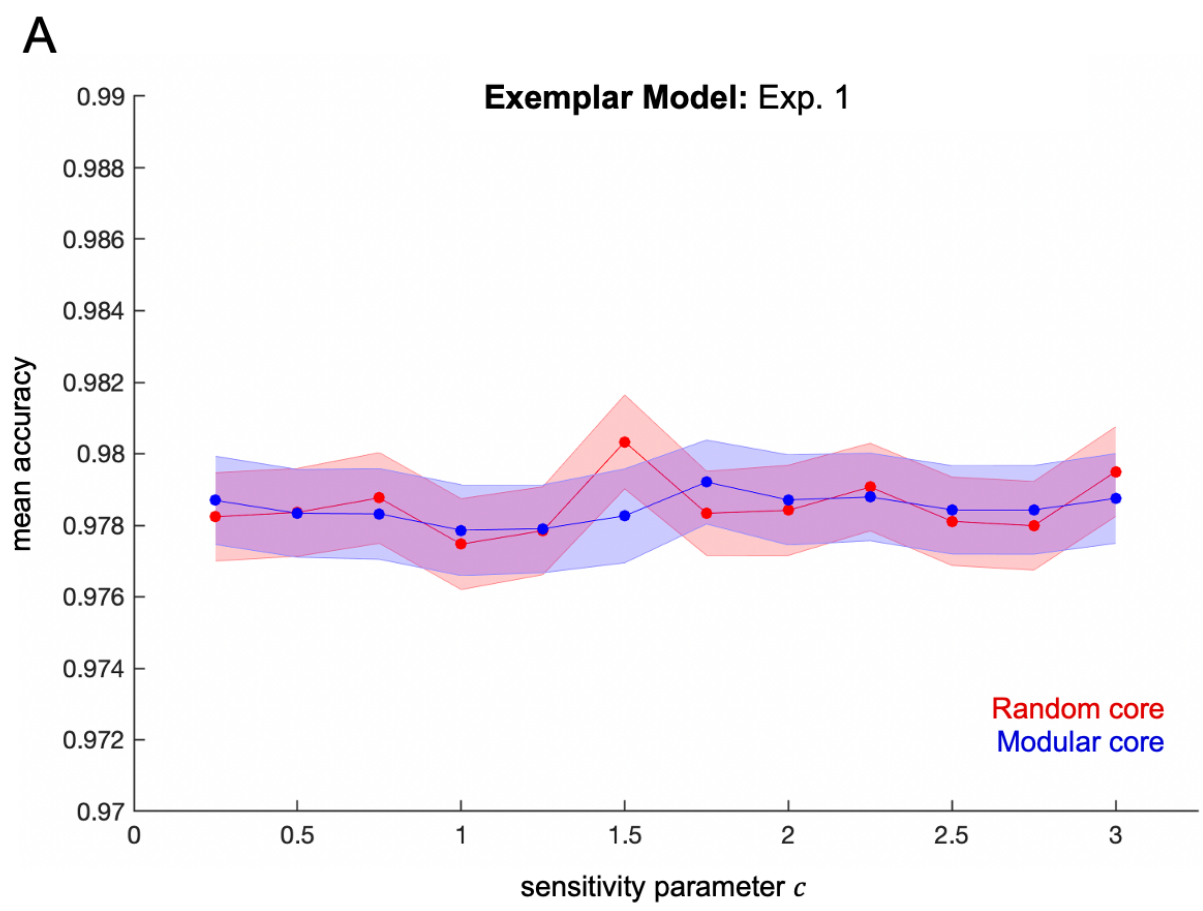

B

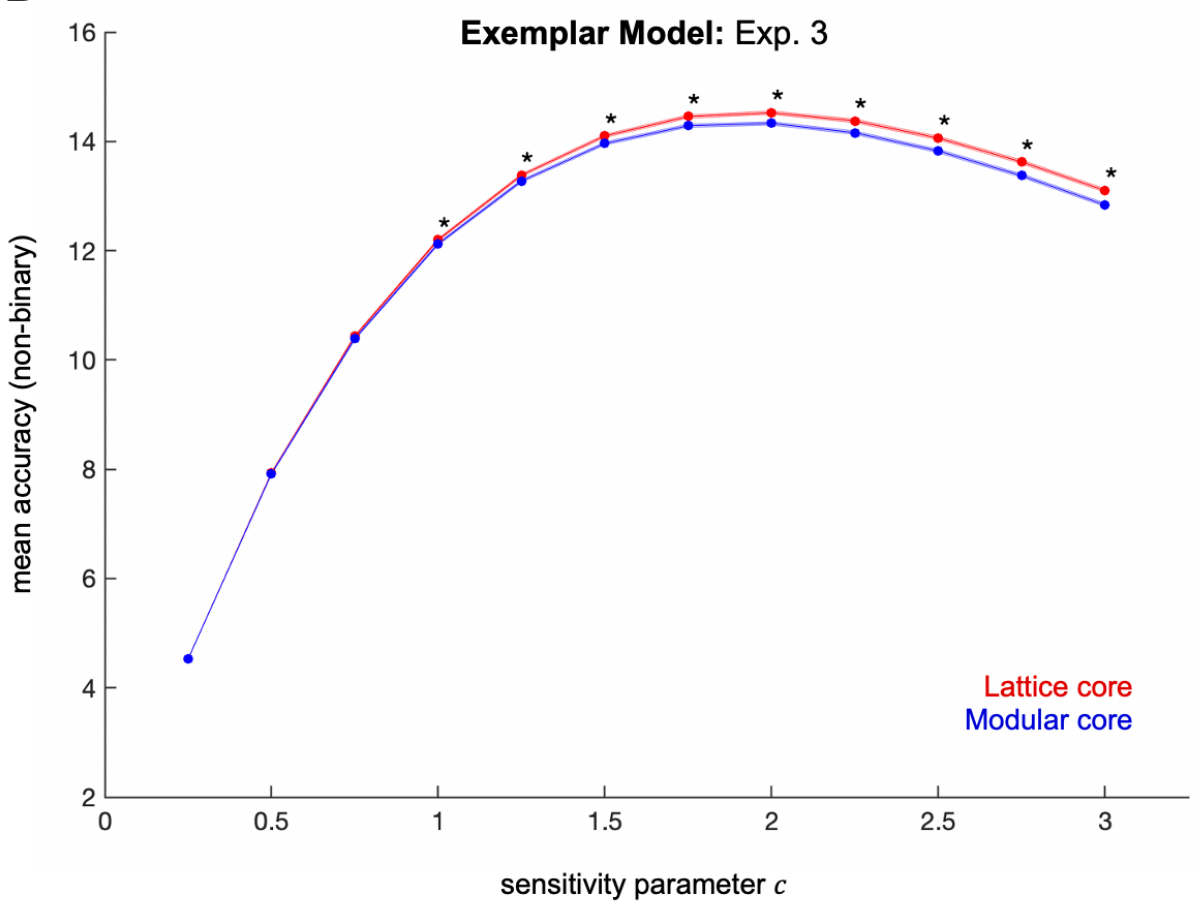

Supplementary Figure 1: Exemplar model performance at different values of the sensitivity parameter $c$, nperm $=500$. (A) In simulations of the missing feature task in Exp. 1, the exemplar model did not predict any difference in core structure accuracy between Random and Modular categories. (B) In simulations of the SL and 2AFC tasks in Exp. 3, the exemplar model did not predict increased accuracy for Modular relative to Lattice core structure trials. Using the non-binary accuracy measure, the exemplar model actually predicted the opposite pattern of results at a range of $c$ values. 